\title{
ISOMETRIC DILATIONS OF NON-COMMUTING FINITE RANK N-TUPLES
}

\author{
KENNETH R. DAVIDSON, DAVID W. KRIBS, AND MIRON E. SHPIGEL
}

\begin{abstract}
A contractive $n$-tuple $A=\left(A_{1}, \ldots, A_{n}\right)$ has a minimal joint isometric dilation $S=\left(S_{1}, \ldots, S_{n}\right)$ where the $S_{i}$ 's are isometries with pairwise orthogonal ranges. This determines a representation of the Cuntz-Toeplitz algebra. When $A$ acts on a finite dimensional space, the wOT-closed nonself-adjoint algebra $\mathfrak{S}$ generated by $S$ is completely described in terms of the properties of $A$. This provides complete unitary invariants for the corresponding representations. In addition, we show that the algebra $\mathfrak{S}$ is always hyper-reflexive. In the last section, we describe similarity invariants. In particular, an $n$-tuple $B$ of $d \times d$ matrices is similar to an irreducible $n$-tuple $A$ if and only if a certain finite set of polynomials vanish on $B$.
\end{abstract}

In $[15,16]$, the first author and David Pitts studied a class of algebras coined free semigroup algebras. These are the WOT-closed (nonself-adjoint) unital operator algebras generated by an $n$-tuple of isometries with pairwise orthogonal ranges. When these ranges span the whole space, the associated norm-closed self-adjoint algebra is a representation of the Cuntz algebra. This nonself-adjoint algebra can contain detailed information about fine unitary invariants of the corresponding $\mathrm{C}^{*}$-algebra representation. Indeed in [15] the set of atomic representations of the Cuntz algebra is completely classified. On the other hand, when the ranges span a proper subspace, the representation contains a multiple of the left regular representation of the free semigroup on $n$ letters. The WOT-closed algebra of the left regular representation is called the non-commutative analytic Toeplitz algebra. This nomenclature is justified by a good analogue of Beurling's Theorem [29, 1, 15], hyper-reflexivity [15] and the relationship [16] between its automorphism group and the group of conformal automorphisms of the ball in $\mathbb{C}^{n}$.

1991 Mathematics Subject Classification. 47L80.

March 10, 1999. Revised May 1, 2000.

The first author was partially supported by an NSERC grant.

The second author was partially supported by NSERC and OGS scholarships. 
The connection with dilation theory derives from a theorem of Frahzo, Bunce and Popescu $[\mathbf{1 9}, \mathbf{1 1}, \mathbf{2 6}]$. If $A=\left(A_{1}, \ldots, A_{n}\right)$ is an $n$-tuple of operators such that $A A^{*}=\sum_{i=1}^{n} A_{i} A_{i}^{*} \leq I$, then there is a unique minimal isometric dilation to isometries $S_{i}$ on a larger space with pairwise orthogonal ranges. Popescu [26] establishes the analogue of Wold's decomposition which splits this into a direct sum of a multiple of the left regular representation and a representation of the Cuntz algebra. Moreover, Popescu [28] obtains the non-commutative analogue of von Neumann's inequality in this context. We mention in passing that there has been recent interest in dilating commuting $n$-tuples as well $[30,3,4]$.

On the other hand, representations of the Cuntz algebra correspond to endomorphisms of $\mathcal{B}(\mathcal{H})[\mathbf{3 1}, \mathbf{2 5}, \mathbf{8}, \mathbf{9}]$. This has created new interest in classifying these representations up to unitary equivalence. The well-known theorem of Glimm [22] shows that this classification is non-smooth because $\mathcal{O}_{n}$ is anti-liminal (or NGCR). Nevertheless, interesting classes of representations do lend themselves to a complete analysis. In [10], Bratteli and Jorgensen introduced a class of representations which turned out to be a special case of the atomic representations classified in [15] using nonself-adjoint techniques. In [9] they introduce a different class associated to finitely correlated states. The reader will see a lot of parallels between their results and ours, though the approach is quite different. In the end, they specialize to the subclass of diagonalizable shifts in order to obtain a classification theorem. In this paper, we obtain good unitary invariants for the class of all of these finitely correlated representations.

The goal of this paper is two-fold. First we wish to understand the structure of the free semigroup algebra generated by the dilation of an $n$-tuple $A$ in terms of information obtained from the $n$-tuple itself (and the algebra it generates). In particular, we seek unitary invariants for the associated $\mathrm{C}^{*}$-algebra representation. Secondly, we wish to determine whether these algebras are reflexive and even hyper-reflexive. In this paper, we focus on the case in which the $n$-tuple $A$ acts on a finite dimensional space. Here we obtain a complete description of the algebra. This enables us to decompose the associated representation as a direct sum of irreducible representations and obtain complete unitary invariants. These algebras all turn out to be hyper-reflexive.

In the last section, we discuss similarity invariants. One of the surprising consequences is a complete invariant for an irreducible $n$-tuple of $d \times d$ matrices up to similarity. An algorithm for determining if two $n$-tuples of matrices are similar is provided by Friedland [21]. But this method rapidly gets complicated. So it is not clear whether it is 
superior to ours. We find that there is a finite set of no more than $1+(n-1) d^{2}$ polynomials $p_{j}$ so that another $n$-tuple $B$ is similar to $A$ if and only if $p_{j}(B)=0$ for all $j$. These polynomials are obtained from a computable set of generators of an ideal of the left regular free semigroup algebra as a right ideal, which amounts to computing an orthonormal basis for a certain subspace. In practice, one only needs generators as a two-sided ideal, and hence the actual number needed is normally smaller.

\section{BACKGROUND}

Let $\mathbb{F}_{n}^{+}$denote the unital free semigroup on $n$ letters $\{1,2, \ldots, n\}$, and let $\mathcal{K}_{n}=\ell^{2}\left(\mathbb{F}_{n}^{+}\right)$denote the Hilbert space with basis $\left\{\xi_{w}: w \in \mathbb{F}_{n}^{+}\right\}$, which is known as $n$-variable Fock space. The left regular representation $\lambda$ of $\mathbb{F}_{n}^{+}$is given by $\lambda(v) \xi_{w}:=L_{v} \xi_{w}=\xi_{v w}$. In particular, the generators of $\mathbb{F}_{n}^{+}$determine isometries $L_{i}$ for $1 \leq i \leq n$ with orthogonal ranges such that $\sum_{i=1}^{n} L_{i} L_{i}^{*}=I-P_{e}$ where $P_{e}=\xi_{e} \xi_{e}^{*}$ is the rank one projection onto the basis vector for the empty word $e$, which is the identity of $\mathbb{F}_{n}^{+}$. The algebra $\mathfrak{L}_{n}$ is the WOT-closed algebra generated by the $n$-tuple $L=\left(L_{1}, \ldots, L_{n}\right)$. See $[15,16,17,23,27,29]$ for detailed information about this algebra.

More generally if $S_{i}, 1 \leq i \leq n$, are isometries with $\sum_{i=1}^{n} S_{i} S_{i}^{*} \leq I$, let $\mathfrak{S}$ denote the unital WOT-closed (nonself-adjoint) algebra generated by them. We denote by $S_{v}$ the isometry $v(S):=v\left(S_{1}, \ldots, S_{n}\right)$ for each $v \in \mathbb{F}_{n}^{+}$. A subspace $\mathcal{W}$ is called wandering for the $n$-tuple $S=\left(S_{1}, \ldots, S_{n}\right)$ provided that the subspaces $S_{v} \mathcal{W}$ are pairwise orthogonal for all $v \in \mathbb{F}_{n}^{+}$. Thus the smallest $\mathfrak{S}$-invariant subspace containing a wandering space $\mathcal{W}$ is $\mathfrak{S}[\mathcal{W}]=\sum_{v \in \mathbb{F}_{n}^{+}}^{\oplus} S_{v} \mathcal{W}$. The restriction of $\mathfrak{S}$ to this subspace is evidently a multiple of the left regular representation algebra $\mathfrak{L}_{n}$, where the multiplicity is given by $\operatorname{dim} \mathcal{W}$. Popescu's Wold decomposition [26] works as follows: the subspace $\mathcal{W}=\operatorname{Ran}\left(I-\sum_{i=1}^{n} S_{i} S_{i}^{*}\right)$ is easily seen to be wandering. Moreover the complement $\mathcal{N}=\mathfrak{S}[\mathcal{W}]^{\perp}$ is also invariant for $\mathfrak{S}$, and the restriction to $\mathcal{N}$ yields isometries $T_{i}=\left.S_{i}\right|_{\mathcal{N}}$ satisfying $\sum_{i=1}^{n} T_{i} T_{i}^{*}=I_{\mathcal{N}}$.

Suppose $A=\left(A_{1}, \ldots, A_{n}\right)$ is an $n$-tuple of operators on a Hilbert space $\mathcal{V}$ such that $A A^{*}=\sum_{i=1}^{n} A_{i} A_{i}^{*} \leq I$. Frahzo [19] (for $n=2$ ), Bunce [11] (for $n<\infty$ ) and Popescu [26] (for $n=\infty$ ) show that there is a joint dilation of the $A_{i}$ to isometries $S_{i}$ on a Hilbert space $\mathcal{H}=\mathcal{V} \oplus \mathcal{K}$ which have pairwise orthogonal ranges. Popescu observes that if this dilation is minimal in the sense that $\mathcal{H}=\operatorname{span}\left\{S_{v} \mathcal{V}: v \in \mathbb{F}_{n}^{+}\right\}$, then the dilation is unique (up to a unitary equivalence which fixes $\mathcal{V}$ ). We will always work with this minimal isometric dilation. 
Popescu also observes [28] that the norm-closed nonself-adjoint algebra $\mathcal{A}_{n}$ spanned by $\left\{L_{w}: w \in \mathbb{F}_{n}^{+}\right\}$is the appropriate non-commutative analogue of the disk algebra for a version of von Neumann's inequality. Namely, if $A$ is a contractive $n$-tuple as above, then $\|p(A)\| \leq\|p(L)\|$ for every non-commuting polynomial in $n$ variables. This is immediate from the dilation theorem and the fact that there is a contractive homomorphism of $\mathcal{E}_{n}$ onto $\mathcal{O}_{n}$, the two possible $\mathrm{C}^{*}$-algebras for the dilation. However, it turns out that this quotient map is completely isometric on $\mathcal{A}_{n}$. So this norm estimate is an equality for any contractive $n$-tuple of isometries. This shows that $\mathcal{O}_{n}$ is the $\mathrm{C}^{*}$-envelope of $\mathcal{A}_{n}$.

This presents a rather precise picture for the norm-closed algebra generated by an $n$-tuple of isometries with orthogonal ranges. However, the WOT-closed algebras can be quite different. They can reflect the fine unitary invariants of the representation. The case $n=1$ is familiar, where the WOT-closed algebra depends on the spectral invariants of the unitary part and the multiplicity of the shift (from the Wold decomposition).

When $\sum_{i=1}^{n} S_{i} S_{i}^{*}=I$, the $\mathrm{C}^{*}$-algebra generated by the isometries $S_{i}$ is the Cuntz algebra $\mathcal{O}_{n}$; and when $\sum_{i=1}^{n} S_{i} S_{i}^{*}<I$, this $\mathrm{C}^{*}$-algebra is $*$-isomorphic to the Cuntz-Toeplitz algebra $\mathcal{E}_{n}$ generated by the left regular representation $\lambda$. This algebra is an extension of the compact operators $\mathfrak{K}$ by $\mathcal{O}_{n}$. We associate to each $n$-tuple $S_{i}$ the representation $\sigma$ of $\mathcal{E}_{n}$ given by $\sigma\left(s_{i}\right)=S_{i}$, where $s_{i}$ are the canonical generators of $\mathcal{E}_{n}$. When $\sum_{i=1}^{n} S_{i} S_{i}^{*}=I$, we may consider this as a representation of $\mathcal{O}_{n}$ instead. Let $\mathfrak{S}_{\sigma}$ denote the WOT-closed non-self-adjoint algebra determined by the representation $\sigma$. One can view the Wold decomposition as the spatial view of the $\mathrm{C}^{*}$-algebra fact that every representation $\sigma$ of $\mathcal{E}_{n}$ splits as a direct sum $\sigma=\lambda^{(\alpha)} \oplus \tau$ of a representation $\lambda^{(\alpha)}$, which is faithful on $\mathfrak{K}$ and thus is a multiple of the identity representation $\lambda$, and a representation $\tau$ which factors through $\mathcal{O}_{n}$.

A representation is called atomic if there is an orthonormal basis $\left\{\xi_{j}\right\}$ which are permuted up to scalars by the generating isometries $S_{i}$. That is, for each $i$ there is an endomorphism $\pi_{i}: \mathbb{N} \rightarrow \mathbb{N}$ and scalars $\lambda_{i, j}$ of modulus 1 such that $S_{i} \xi_{j}=\lambda_{i, j} \xi_{\pi_{i}(j)}$. These representations decompose as a direct integral of irreducible atomic representations [15], and these irreducible atomic representations are of three types. The first is just the left regular representation; which is the only one which does not factor through $\mathcal{O}_{n}$. The second type is a class of inductive limits of the left regular representation, and are classified by an infinite word (up to shift-tail equivalence) that describes the imbeddings. The third type fits into the context of this paper, and so we describe it in more detail. See [15] for a complete description. 
The third type is given by a word $u=i_{1} i_{2} \ldots i_{d}$ in $\mathbb{F}_{n}^{+}$and a scalar $\lambda$ of modulus 1. A finite dimensional space $\mathcal{V}$ of dimension $d$ is formed with a basis $e_{1}, \ldots, e_{d}$. Operators $A_{j}, 1 \leq j \leq n$, are partial isometries given by

$$
\begin{aligned}
& A_{j} e_{k}=\delta_{j i_{k}} e_{k+1} \quad \text { for } \quad 1 \leq k<d \\
& A_{j} e_{d}=\lambda \delta_{j i_{d}} e_{1} .
\end{aligned}
$$

The minimal isometric dilation of this $n$-tuple yields isometries $S_{j}$ acting on a space $\mathcal{H}=\mathcal{V} \oplus \mathcal{K}$. The isometry $S_{i_{k}}$ maps $e_{k}$ to $e_{k+1}$ (or $\lambda e_{1}$ when $k=d$ ) and the other $n-1$ isometries send $e_{k}$ to pairwise orthogonal vectors which are all wandering vectors for $\mathfrak{S}$. Thus $\mathcal{K}=\mathcal{V}^{\perp}$ is determined by a wandering space $\mathcal{W}$ of dimension $d(n-1)$, and therefore $\mathcal{K}=\mathfrak{S}[\mathcal{W}] \simeq \mathcal{K}_{n}^{(d(n-1))}$. The associated representation $\sigma_{u, \lambda}$ is irreducible precisely when the word $u$ is primitive, meaning that it is not a power of a smaller word. In this case, $\mathfrak{S}$ can be completely described as the sum of $\mathcal{B}(\mathcal{H}) P_{\mathcal{V}}$ and a multiple of $\mathfrak{L}_{n}$ acting on $\mathcal{K}$ via its identification with $\mathcal{K}_{n}^{(d(n-1))}$. The invariant subspaces of this algebra are readily described, and it turns out to be hyper-reflexive. (See below).

For the future, we wish to name the type of algebra which occurs here. Let $\mathfrak{B}_{n, d}$ denote the wOT-closed algebra on a Hilbert space $\mathcal{H}=$ $\mathcal{V} \oplus \mathcal{K}_{n}^{(d(n-1))}$ where $\operatorname{dim} \mathcal{V}=d$ given by

$$
\mathfrak{B}_{n, d}=\mathcal{B}(\mathcal{H}) P_{\mathcal{V}}+\left(0_{\mathcal{V}} \oplus \mathfrak{L}_{n}^{(d(n-1))}\right) .
$$

Another class of representations which have been studied are the finitely correlated representations [9]. A representation of $\mathcal{O}_{n}$ is finitely correlated if there is a finite dimensional cyclic subspace $\mathcal{V}$ which is invariant for each $S_{i}^{*}$. Likewise, a finite correlated state is a state $\varphi$ such that in the GNS construction, the invariant subspace for the $S_{i}^{*}$ 's generated by the cyclic vector $\xi_{\varphi}$ is finite dimensional. It is evident that these representations are exactly those which we will study from the viewpoint of dilation theory. In this paper, we will obtain a complete classification of these representations up to unitary equivalence. We will explain later how our classification relates to the work of Bratteli and Jorgensen.

If $\mathfrak{A}$ is an algebra of operators, Lat $\mathfrak{A}$ denotes the lattice of all $\mathfrak{A}$ invariant subspaces. And if $\mathcal{L}$ is a lattice of subspaces, $\operatorname{Alg} \mathcal{L}$ denotes the WOT-closed unital algebra of all operators which leave each element of $\mathcal{L}$ invariant. The algebra $\mathfrak{A}$ is reflexive if it equals $\mathrm{Alg}$ Lat $\mathfrak{A}$. For each reflexive algebra, there is a quantitative measure of the distance 
to $\mathfrak{A}$ given by

$$
\beta_{\mathfrak{A}}(T)=\sup _{L \in \mathcal{L}}\left\|P_{L}^{\perp} T P_{L}\right\| .
$$

It is easily seen that $\beta_{\mathfrak{A}}(T) \leq \operatorname{dist}(T, \mathfrak{A})$. The algebra is called hyperreflexive if there is a constant $C$ such that $\operatorname{dist}(T, \mathfrak{A}) \leq C \beta_{\mathfrak{A}}(T)$. The optimal $C$, if it is finite, is called the distance constant for $\mathfrak{A}$.

The list of algebras known to be hyper-reflexive is rather short. Arveson [2] showed that nest algebras have distance constant 1 , so that equality is achieved. Christensen [12] showed that AF von Neumann algebras have distance constant at most 4 . Concerning the algebras studied in this paper, the first author [14] showed that the analytic Toeplitz algebra has distance constant at most 19; and with Pitts [15], that all atomic free semigroup algebras where shown to have distance constant at most 51. The worst case for these estimates was the algebra $\mathfrak{L}_{n}$. However a recent general result of Bercovici [6] applies to show that $\mathfrak{L}_{n}$ actually has a distance constant no greater than 3 .

\section{MAin Results}

In this paper, we generally take $n$ to be a finite integer with $n \geq 2$. However, Popescu's version of the dilation theorem is valid for $n=\infty$, as are the results of $[\mathbf{1 5}, \mathbf{1 6}]$ on the structure of $\mathfrak{L}_{n}$ which we shall use. So the results of this paper go through for $n=\infty$ with only a few minor changes in notation, not in substance. For ease of presentation, we will write this paper as though $n$ were finite, and let the interested reader interpolate the $n=\infty$ case.

Consider a contractive $n$-tuple $A=\left(A_{1}, \ldots, A_{n}\right)$ acting on a finite dimensional space $\mathcal{V}$ of dimension $d$; i.e. $\sum_{i=1}^{n} A_{i} A_{i}^{*} \leq I$. The FrahzoBunce-Popescu minimal dilation yields isometries $S_{i}$ acting on a larger space $\mathcal{H}$. We let $\mathfrak{A}$ denote the algebra generated by the $A_{i}$ 's, and let $\mathfrak{S}$ be the WOT-closed algebra generated by the $S_{i}$ 's. We will make important use of an associated completely positive contractive map on $\mathcal{B}(\mathcal{V})$ given by

$$
\Phi(X)=\sum_{i=1}^{n} A_{i} X A_{i}^{*} .
$$

The operator $\Phi^{\infty}(I):=\lim _{k \rightarrow \infty} \Phi^{k}(I)$ will also be useful.

The first fairly easy observation is that the dilation is of Cuntz type $\left(\sum_{i=1}^{n} S_{i} S_{i}^{*}=I\right)$ if and only if $\sum_{i=1}^{n} A_{i} A_{i}^{*}=I$; or equivalently $\Phi(I)=I$. In general, we define the pure rank of $\mathfrak{S}$ to be the multiplicity of the left regular representation in the Wold decomposition of $\mathfrak{S}$. This is the dimension of the wandering space $\mathcal{W}=\operatorname{Ran}\left(I-\sum_{i=1}^{n} S_{i} S_{i}^{*}\right)$. Simple examples show that this wandering space need not be contained in $\mathcal{V}$, 
and that even when this pure rank is one, the pure part may have large intersection with $\mathcal{V}$. Nevertheless, it turns out that this pure rank may be easily computed as

$$
\operatorname{pure} \operatorname{rank}(\mathfrak{S})=\operatorname{rank}(I-\Phi(I))=\operatorname{rank}\left(I-\sum_{i=1}^{n} A_{i} A_{i}^{*}\right) .
$$

The irreducible summands of Cuntz type are determined by the minimal $\mathfrak{A}^{*}$-invariant subspaces $\mathcal{M}$ of $\mathcal{V}$ on which $\left.\sum_{i=1}^{n} A_{i} A_{i}^{*}\right|_{\mathcal{M}}=I_{\mathcal{M}}$. Such a subspace generates an invariant subspace $\mathcal{H}_{\mathcal{M}}=\mathfrak{S}[\mathcal{M}]$ for $\mathfrak{S}$ which is necessarily reducing. The restriction $\left.\mathfrak{S}\right|_{\mathcal{H}_{\mathcal{M}}}$ of $\mathfrak{S}$ to this subspace is isomorphic to the algebra $\mathfrak{B}_{n, m}$, where $m=\operatorname{dim} \mathcal{M}$, described in the Background section. A crucial feature is that the projection $P_{\mathcal{M}}$ belongs to this algebra. This makes it possible to show that the restriction of the $n$-tuple $A$ to $\mathcal{M}$ is a unitary invariant for the dilation.

The subspace $\widetilde{\mathcal{V}}$ spanned by all the minimal $\mathfrak{A}^{*}$-invariant subspaces of this type completely determines the Cuntz part of the dilation. The restriction of $\mathfrak{A}^{*}$ to $\widetilde{\mathcal{V}}$ is a finite dimensional $\mathrm{C}^{*}$-algebra. The well-known invariants for a finite dimensional $\mathrm{C}^{*}$-algebra allow one to compute the multiplicities of each irreducible subrepresentation. In general, this information may be used to completely decompose the representation into a direct sum of finitely many irreducible representations of the types given above. This yields complete unitary invariants: the pure rank and the unitary equivalence class of the restriction of $A^{*}$ to $\widetilde{\mathcal{V}}$.

For example, one can show that $\mathfrak{S}$ is irreducible if and only if either (1) $\operatorname{rank}(I-\Phi(I))=1$ and $\Phi^{\infty}(I)=0$, the pure case, or (2) $\{X: \Phi(X)=X\}=\mathbb{C} I$, the Cuntz case.

The algebras $\mathfrak{L}_{n}$ and $\mathfrak{B}_{n, d}$ were shown to be hyper-reflexive in [15]. This analysis can be used to show that all of these algebras $\mathfrak{S}$ determined by a finite rank $n$-tuple are hyper-reflexive. The constant 51 of that paper may be improved to 5 using recent results of Bercovici [6] which show that the distance constant for $\mathfrak{L}_{n}$ is at most 3 .

Then we turn our attention to similarity. If two contractive $n$-tuples are similar, it follows that their Cuntz parts are unitarily equivalent. However, the pure rank can change. Indeed, this rank can be 0 in one case and non-zero in a similar $n$-tuple.

The major interest lies in the pure case. In this case, the algebra $\mathfrak{S}$ is unitarily equivalent to a multiple of $\mathfrak{L}_{n}$, and thus completely isometrically isomorphic and weak-* homeomorphic to $\mathfrak{L}_{n}$. The compression $\Phi_{A}$ of $\mathfrak{S}$ to $\mathcal{V}$ is thus a weak-* continuous representation of $\mathfrak{L}_{n}$. The study of these representations was initiated in [16]. The kernel of such a representation is a WOT-closed ideal. A WOT-closed ideal $\mathfrak{J}$ of $\mathfrak{L}_{n}$ 
is determined [16, Theorem 2.1] by its range $\mathcal{M}=\overline{\mathfrak{J} \mathcal{K}_{n}}$, which is a subspace invariant for both $\mathfrak{L}_{n}$ and its commutant $\mathfrak{R}_{n}$. Thus we consider the associated representation of $\mathfrak{L}_{n}$ obtained as restriction to $\mathcal{M}^{\perp}$. This has the same kernel $\mathfrak{J}$. In the case of an irreducible $n$-tuple, the minimal $\mathfrak{L}_{n}^{*}$-invariant subspaces of $\mathcal{M}^{\perp}$ yield all of the $n$-tuples similar to $A$ which have pure rank 1 . These are the extreme points of all such representations in the sense that $A$ can be recovered as a $\mathrm{C}^{*}$-convex combination of them.

In particular, it follows that two irreducible $n$-tuples of matrices are similar if and only if the induced representations of $\mathfrak{L}_{n}$ have the same kernel. The range space $\mathcal{M}$ of $\mathfrak{J}=\operatorname{ker} \Phi_{A}$ has a wandering space of dimension $1+(n-1) d^{2}$. A basis for this wandering space yields a finite set of generators for $\mathfrak{J}$ as a WOT-closed right ideal. They determine a corresponding finite set of isometries $X_{j}$ in $\mathfrak{L}_{n}$ with the property that another contractive $n$-tuple of $d \times d$ matrices $B$ is similar to $A$ if and only if $\Phi_{B}\left(X_{j}\right)=0$ for $1 \leq j \leq 1+(n-1) d^{2}$. Since one merely requires generators for $\mathfrak{J}$ as a two-sided ideal, normally this number of tests can be reduced. The set of isometries are canonical, but they are generally not polynomials. A set of polynomial invariants can be obtained by an approximation argument.

\section{WANDERING SUBSPACES}

Let $\mathcal{V}$ be a $d$-dimensional space (possibly infinite), and let $A_{1}, \ldots, A_{n}$ be an $n$-tuple of operators in $\mathcal{B}(\mathcal{V})$ such that $\sum_{i=1}^{n} A_{i} A_{i}^{*} \leq I$. The Frahzo-Bunce-Popescu minimal dilation yields isometries $S_{i}$ on a larger space $\mathcal{H}$. Let $P_{\mathcal{V}}$ denote the projection of $\mathcal{H}$ onto $\mathcal{V}$. We let $\mathfrak{A}$ denote the algebra generated by the $A_{i}$ 's and $\mathfrak{S}$ be the wOT-closed algebra generated by the $S_{i}$ 's. We first identify $\mathcal{V}^{\perp}$.

Lemma 3.1. The subspace $\mathcal{W}=\left(\mathcal{V}+\sum_{i=1}^{n} S_{i} \mathcal{V}\right) \ominus \mathcal{V}$ is a wandering subspace for $S$, and $\sum_{v \in \mathbb{F}_{n}^{+}}^{\oplus} S_{v} \mathcal{W}=\mathcal{V}^{\perp}$.

Proof. $\mathcal{W}$ is contained in $\mathcal{V}^{\perp}$, which is invariant for $S$. Thus $S_{u} \mathcal{W}$ is orthogonal to $\mathcal{V}$ for every word $u \in \mathbb{F}_{n}^{+}$. Consequently, when $|u| \geq 1$, $S_{u} \mathcal{W}$ is also orthogonal to $S_{j} \mathcal{V}, 1 \leq j \leq n$. It follows that $S_{u} \mathcal{W}$ is orthogonal to $\mathcal{V}+\sum_{i=1}^{n} S_{i} \mathcal{V}$, which contains $\mathcal{W}$. Therefore $\mathcal{W}$ is wandering. Minimality ensures that

$$
\mathcal{H}=\operatorname{span}\left\{S_{u} \mathcal{V}: u \in \mathbb{F}_{n}^{+}\right\}=\operatorname{span}\left\{\mathcal{V}, S_{u} \mathcal{W}: u \in \mathbb{F}_{n}^{+}\right\}
$$

Since $\mathcal{W}$ lies in the invariant subspace $\mathcal{V}^{\perp}$, this can only occur because $\sum_{v \in \mathbb{F}_{n}^{+}}^{\oplus} S_{v} \mathcal{W}=\mathcal{V}^{\perp}$. 
Thus $\mathcal{K}=\mathcal{V}^{\perp}$ is unitarily equivalent to a multiple $\mathcal{K}_{n}^{(\alpha)}$ of Fock space, where $\alpha=\operatorname{dim} \mathcal{W}$, and $\left.S_{i}\right|_{\mathcal{K}} \simeq L_{i}^{(\alpha)}$. Hence decomposing $\mathcal{H}=\mathcal{V} \oplus \mathcal{K}$, we may write each $S_{i}$ as a matrix $S_{i}=\left[\begin{array}{cc}A_{i} & 0 \\ X_{i} & L_{i}^{(\alpha)}\end{array}\right]$.

Remark 3.2. The range of $\sum_{i=1}^{n} S_{i} S_{i}^{*}$ includes $\sum_{i=1}^{n} S_{i} \mathcal{V}^{\perp}=(\mathcal{V}+\mathcal{W})^{\perp}$ as well as $\sum_{i=1}^{n} S_{i} \mathcal{V}$. Hence $\sum_{i=1}^{n} S_{i} S_{i}^{*}=I$ if and only if $\sum_{i=1}^{n} S_{i} \mathcal{V}$ contains $\mathcal{V}$. Since $\mathcal{V}$ is invariant for $S_{i}^{*}$ and $\left.S_{i}^{*}\right|_{\mathcal{V}}=A_{i}^{*}$,

$$
\sum_{i=1}^{n} A_{i} A_{i}^{*}=\left.\sum_{i=1}^{n} P_{\mathcal{V}} S_{i} P_{\mathcal{V}} S_{i}^{*}\right|_{\mathcal{V}}=\left.P_{\mathcal{V}} \sum_{i=1}^{n} S_{i} S_{i}^{*}\right|_{\mathcal{V}}
$$

Therefore $\sum_{i=1}^{n} S_{i} S_{i}^{*}=I$ if and only if its range contains $\mathcal{V}$ if and only if $\sum_{i=1}^{n} A_{i} A_{i}^{*}=I_{\mathcal{V}}$.

Let $d=\operatorname{dim} \mathcal{V}$ be finite, and let $\alpha=\operatorname{dim} \mathcal{W}$. Then $\alpha$ can be as large as $n d$ and as small as $(n-1) d$. This is easily seen since $\sum_{i=1}^{n} S_{i} \mathcal{V}$ is an orthogonal direct sum and thus has dimension $n d$, so that $\mathcal{W}=$ $\left(\mathcal{V}+\sum_{i=1}^{n} S_{i} \mathcal{V}\right) \ominus \mathcal{V}$ can have no larger dimension than $n d$, and is at least $(n-1) d$.

When $\sum_{i=1}^{n} A_{i} A_{i}^{*}=I_{\mathcal{V}}$, we showed above that $\sum_{i=1}^{n} S_{i} S_{i}^{*}=I$. Then

$$
\mathcal{V}=\sum_{i=1}^{n} S_{i} S_{i}^{*} \mathcal{V}=\sum_{i=1}^{n} S_{i} A_{i}^{*} \mathcal{V} \subset \sum_{i=1}^{n} S_{i} \mathcal{V}
$$

Hence $\mathcal{W}=\left(\sum_{i=1}^{n} S_{i} \mathcal{V}\right) \ominus \mathcal{V}$ has dimension $(n-1) d$.

The case $\operatorname{dim} \mathcal{W}=n d$ occurs, for example, if $A_{i}=0$ for $1 \leq i \leq n$. The minimal dilation is just $L_{i}^{(d)}$. Indeed, if $x, y \in \mathcal{V}$, then

$$
\left(S_{i} x, y\right)=\left(x, S_{i}^{*} y\right)=\left(x, A_{i}^{*} y\right)=0 \quad \text { for } \quad 1 \leq i \leq n .
$$

Thus $\mathcal{V}$ is orthogonal to $\sum_{i=1}^{n} S_{i} \mathcal{V}$. Therefore $\mathcal{W}=\sum_{i=1}^{n} S_{i} \mathcal{V}$ has dimension $n d$.

It is easy to combine these examples to obtain any integer in between.

The $n$-tuple of isometries $S$ is called pure if it is unitarily equivalent to a multiple of the left regular representation. Bunce [11] shows that whenever $\|A\|<1$, the dilation $S$ is pure. Popescu [26] shows that the dilation is pure if and only if $\underset{k \rightarrow \infty}{\mathrm{WOT}-\lim } \sum_{|v|=k} A_{v} A_{v}^{*}=0$.

Lemma 3.1 shows that beginning with an $n$-tuple, we will always obtain wandering vectors except when the $A_{i}$ 's already are isometries and $\sum_{i=1}^{n} A_{i} A_{i}^{*}=I$, in which case the dilation is just the $A_{i}$ 's themselves. When there are wandering vectors, each generates a subspace $\mathcal{M}$ on which the isometries $S_{i}$ are unitarily equivalent to the left regular representation. In particular, the non-* algebra $\mathfrak{S}$ that they generate is very non-self-adjoint. In fact, a strong converse to this exists. Recall 
that an algebra is reductive if all of its invariant subspaces have invariant (orthogonal) complements; and it is transitive if it has no proper invariant subspaces at all. It is an open problem equivalent to the transitive algebra variant of the invariant subspace problem [18] whether every WOT-closed reductive algebra is self-adjoint.

Lemma 3.3. Let $\mathfrak{S}$ be a free semigroup algebra. Then either $\mathfrak{S}$ has a wandering vector or $\mathfrak{S}$ is reductive. If the latter is possible, then transitive free semigroup algebras exist.

Proof. Suppose that $\mathfrak{S}$ has no wandering vectors. Let $\mathcal{M}$ be an invariant subspace for $\mathfrak{S}$. Then $\sum_{i=1}^{n} S_{i} \mathcal{M}$ must equal $\mathcal{M}$; for otherwise $\mathcal{M} \ominus \sum_{i=1}^{n} S_{i} \mathcal{M}$ is wandering. Thus $\sum_{i=1}^{n} S_{i} \mathcal{M}^{\perp}=\mathcal{M}^{\perp}$, so that $\mathcal{M}^{\perp}$ is also invariant. Whence $\mathfrak{S}$ is reductive.

Now we invoke the direct integral theory for non-self-adjoint operator algebras due to Azoff, Fong and Gilfeather [5, Theorem 4.1]. Let $\mathfrak{M}$ be any masa in the commutant of $\mathfrak{S}$. They show that $\mathfrak{S}$ may be decomposed with respect to $\mathfrak{M}$ as an integral of algebras which are transitive almost everywhere. The isometries $S_{i}$ decompose as an integral of operators which are isometries almost everywhere as well. In particular, transitive algebras generated by isometries with orthogonal ranges would exist.

At this point, we do not know if there are transitive free semigroup algebras. The motivation for suspecting that there may be comes from the case $n=1$. A unitary operator is reductive unless Lebesgue measure on the whole circle is absolutely continuous with respect to the spectral measure of the unitary [35]. This is the case for 'most' unitaries.

We will frequently construct reducing subspaces of $\mathfrak{S}$ from $\mathfrak{A}^{*}$-invariant subspaces. This procedure preserves orthogonality as well.

Lemma 3.4. Suppose that $\mathcal{V}$ contains an $\mathfrak{A}^{*}$-invariant subspace $\mathcal{V}_{1}$ Then $\mathcal{H}_{1}=\mathfrak{S}\left[\mathcal{V}_{1}\right]$ reduces $\mathfrak{S}$.

If $\mathcal{V}$ contains a pair of orthogonal $\mathfrak{A}^{*}$-invariant subspaces $\mathcal{V}_{1}$ and $\mathcal{V}_{2}$, then $\mathcal{H}_{j}=\mathfrak{S}\left[\mathcal{V}_{j}\right]$ for $j=1,2$ are mutually orthogonal.

If in addition $\mathcal{V}=\mathcal{V}_{1} \oplus \mathcal{V}_{2}$, then $\mathcal{H}$ decomposes as $\mathcal{H}_{1} \oplus \mathcal{H}_{2}$ and $\mathcal{H}_{j} \cap \mathcal{V}=\mathcal{V}_{j}$ for $j=1,2$.

Proof. Since $\mathcal{V}_{1}$ is invariant for $A_{i}^{*}$, it is also invariant for $S_{i}^{*}$. The $\mathfrak{S}$ invariant subspace $\mathcal{H}_{1}=\mathfrak{S}\left[V_{1}\right]$ is spanned by vectors of the form $S_{w} x$ where $x \in \mathcal{V}_{1}$ and $w \in \mathbb{F}_{n}^{+}$. Notice that $S_{i}^{*} S_{w} x$ equals $S_{w^{\prime}} x$ if $w=i w^{\prime}$, 0 if $w=i^{\prime} w^{\prime}$ for some $i^{\prime} \neq i$, and $S_{i}^{*} x$ if $w=e$. Since $\mathcal{V}_{1}$ is invariant for $\mathfrak{S}^{*}$, each of these possibilities belongs to $\mathcal{H}_{1}$. Thus $\mathcal{H}_{1}$ reduces $\mathfrak{S}$. 
Likewise, if $\mathcal{V}_{1}$ and $\mathcal{V}_{2}$ are orthogonal $\mathfrak{A}^{*}$-invariant subspaces, it follows that $\mathcal{H}_{1}$ and $\mathcal{H}_{2}$ are orthogonal. For if $v_{j} \in \mathcal{V}_{j}$, the inner product $\left(S_{u} v_{1}, S_{w} v_{2}\right)$ can be reduced by cancellation of isometries until either $u$ or $w$ is the identity element. Then, for example when $w=e$,

$$
\left(S_{u} v_{1}, v_{2}\right)=\left(v_{1}, S_{u}^{*} v_{2}\right)=0
$$

by the $\mathfrak{A}^{*}$-invariance of $\mathcal{V}_{2}$ and orthogonality.

Now suppose that $\mathcal{V}=\mathcal{V}_{1} \oplus \mathcal{V}_{2}$. Since $\mathcal{H}_{1}$ contains $\mathcal{V}_{1}$ and is orthogonal to $\mathcal{V}_{2}$, it follows that $\mathcal{H}_{1} \cap \mathcal{V}=\mathcal{V}_{1}$. Finally, $\mathcal{H}_{1} \oplus \mathcal{H}_{2}$ is an $\mathfrak{S}$-reducing subspace containing $\mathcal{V}$, so it is all of $\mathcal{H}$ by the minimality of the dilation.

\section{Finite Dimensional N-TUples}

Now let us specialize to the case when $\mathcal{V}$ is finite-dimensional. In general, we can decompose the $S_{i}$ into a pure part and Cuntz part. Let $\mathcal{X}$ be the range of $I-\sum_{i=1}^{n} S_{i} S_{i}^{*}$, which is the wandering space for the reducing subspace $\mathcal{H}_{p}=\sum_{v \in \mathbb{F}_{n}^{+}}^{\oplus} S_{v} \mathcal{X}$. The restriction of the $S_{i}$ to this space yields a multiple of the left regular representation, where the multiplicity is $\operatorname{dim} \mathcal{X}$. We call this quantity the pure rank of the representation. On the complement $\mathcal{H}_{c}=\mathcal{H}_{p}^{\perp}$, the restrictions of $S_{i}$ yield a representation of the Cuntz algebra. Let $P_{p}$ and $P_{c}$ denote the projections onto $\mathcal{H}_{p}$ and $\mathcal{H}_{c}$ respectively. It is important to note that the projection $P_{p}$ does not commute with $P_{\mathcal{V}}$ in general. So we will obtain a method of computing this pure rank directly from the $A_{i}$ 's.

The key technical tool in our analysis shows that $\mathcal{H}_{c}$ is determined by $\mathcal{V}_{c}:=\mathcal{H}_{c} \cap \mathcal{V}$. This is not the case for $\mathcal{H}_{p}$. Let $R_{k}$ denote the projection onto $\sum_{|v|=k}^{\oplus} S_{v} \mathcal{W}$, where $\mathcal{W}=\left(\mathcal{V}+\sum_{i=1}^{n} S_{i} \mathcal{V}\right) \ominus \mathcal{V}$; and $Q_{k}=\sum_{j \geq k} R_{j}$. Notice that

$$
Q_{k}=\sum_{|w|=k} S_{w} P_{\mathcal{V}}^{\perp} S_{w}^{*}
$$

On any $\mathfrak{S}$-invariant subspace $\mathcal{M}$ on which the restrictions $T_{i}$ of $S_{i}$ are pure, one has for every $x \in \mathcal{M}$

$$
\lim _{k \rightarrow \infty} \sum_{|w|=k}\left\|P_{\mathcal{M}} S_{w}^{*} x\right\|^{2}=\lim _{k \rightarrow \infty} \sum_{|w|=k}\left\|T_{w}^{*} x\right\|^{2}=0 .
$$

In particular, this applies to $\mathcal{H}_{p}$ and $\mathcal{V}^{\perp}$. While for $x \in \mathcal{H}_{c}$, one has

$$
\sum_{|w|=k}\left\|S_{w}^{*} x\right\|^{2}=\|x\|^{2} \quad \text { for all } \quad k \geq 0 .
$$


Key Technical Lemma 4.1. Suppose that $\mathcal{H}_{1}$ is a reducing subspace for $\mathfrak{S}$ contained in $\mathcal{H}_{c}$. Let $x$ be a vector such that $P_{\mathcal{H}_{1}} x \neq 0$. Then the subspace $\mathcal{M}=\mathfrak{S}^{*}[x]$ contains a vector $v$ in $\mathcal{M} \cap \mathcal{V}_{c}$ with $P_{\mathcal{H}_{1}} v \neq 0$.

Proof. Let $P_{1}$ denote the projection of $\mathcal{H}$ onto $\mathcal{H}_{1}$. Fix $\varepsilon>0$; and let $x_{1}=P_{1} x$. By applying the preceding remarks to both $\mathcal{V}^{\perp}$ and $\mathcal{H}_{p}$, we may choose an integer $k$ sufficiently large that

$$
\begin{aligned}
& \sum_{|w|=k}\left\|P_{\mathcal{V}}^{\perp} S_{w}^{*} x\right\|^{2}=\left\|Q_{k} x\right\|^{2}<\varepsilon^{2} \\
& \sum_{|w|=k}\left\|P_{\mathcal{V}}^{\perp} S_{w}^{*} x_{1}\right\|^{2}=\left\|Q_{k} x_{1}\right\|^{2}<\varepsilon^{2}
\end{aligned}
$$

and

$$
\sum_{|w|=k}\left\|P_{p} S_{w}^{*} x\right\|^{2}=\sum_{|w|=k}\left\|S_{w}^{*} P_{p} x\right\|^{2}<\varepsilon^{2} .
$$

Since $\sum_{|w|=k} S_{w} S_{w}^{*} P_{1}=P_{1}$,

$$
\begin{aligned}
\sum_{|w|=k}\left\|P_{\mathcal{V}} S_{w}^{*} x_{1}\right\|^{2} & =\sum_{|w|=k}\left(\left\|S_{w}^{*} x_{1}\right\|^{2}-\left\|P_{\mathcal{V}}^{\perp} S_{w}^{*} x_{1}\right\|^{2}\right) \\
& =\left\|x_{1}\right\|^{2}-\left\|Q_{k} x_{1}\right\|^{2}>\left\|x_{1}\right\|^{2}-\varepsilon^{2} .
\end{aligned}
$$

Let $\mathcal{E}_{1}$ denote the set of words $w$ of length $k$ such that

$$
\left\|P_{\mathcal{V}} S_{w}^{*} x_{1}\right\|^{2}>\varepsilon^{-1}\left\|P_{\mathcal{V}}^{\perp} S_{w}^{*} x\right\|^{2} .
$$

Likewise let $\mathcal{E}_{2}$ denote the set of words $w$ of length $k$ such that

$$
\left\|P_{\mathcal{V}} S_{w}^{*} x_{1}\right\|^{2}>\varepsilon^{-1}\left\|P_{p} S_{w}^{*} x\right\|^{2} .
$$

The set $\mathcal{E}_{1} \cap \mathcal{E}_{2}$ is relatively large in the sense that

$$
\begin{gathered}
\sum_{w \in \mathcal{E}_{1} \cap \mathcal{E}_{2}}\left\|P_{\mathcal{V}} S_{w}^{*} x_{1}\right\|^{2}>\left\|x_{1}\right\|^{2}-\varepsilon^{2}-\sum_{w \notin \mathcal{E}_{1}}\left\|P_{\mathcal{V}} S_{w}^{*} x_{1}\right\|^{2}-\sum_{w \notin \mathcal{E}_{2}}\left\|P_{\mathcal{V}} S_{w}^{*} x_{1}\right\|^{2} \\
>\left\|x_{1}\right\|^{2}-\varepsilon^{2}-\sum_{w \notin \mathcal{E}_{1}} \varepsilon^{-1}\left\|P_{\mathcal{V}}^{\perp} S_{w}^{*} x\right\|^{2}-\sum_{w \notin \mathcal{E}_{2}} \varepsilon^{-1}\left\|P_{p} S_{w}^{*} x\right\|^{2} \\
>\left\|x_{1}\right\|^{2}-\varepsilon^{2}-\varepsilon-\varepsilon>\left\|x_{1}\right\|^{2} / 4
\end{gathered}
$$

for small $\varepsilon$. Now we also have $\sum_{w \in \mathcal{E}_{1} \cap \mathcal{E}_{2}}\left\|P_{\mathcal{V}} S_{w}^{*} x\right\|^{2} \leq\|x\|^{2}$. Therefore there is a word $w$ in $\mathcal{E}_{1} \cap \mathcal{E}_{2}$ such that

$$
\left\|P_{\mathcal{V}} S_{w}^{*} x_{1}\right\|>\frac{\left\|x_{1}\right\|}{2\|x\|}\left\|P_{\mathcal{V}} S_{w}^{*} x\right\|
$$


In this way, construct a sequence of words $w_{k}$ corresponding to $\varepsilon_{k}=$ $1 / k$. Hence define unit vectors $y_{k}=S_{w_{k}}^{*} x /\left\|S_{w_{k}}^{*} x\right\|$ with the properties that

$$
\lim _{k \rightarrow \infty}\left\|P_{\mathcal{V}}^{\perp} y_{k}\right\| \leq \lim _{k \rightarrow \infty} \frac{1}{\sqrt{k}} \frac{\left\|P_{\mathcal{V}} S_{w_{k}}^{*} P_{1} x\right\|}{\left\|S_{w_{k}}^{*} x\right\|}=\lim _{k \rightarrow \infty} \frac{1}{\sqrt{k}} \frac{\left\|P_{\mathcal{V}} P_{1} S_{w_{k}}^{*} x\right\|}{\left\|S_{w_{k}}^{*} x\right\|}=0 .
$$

Similarly,

$$
\lim _{k \rightarrow \infty}\left\|P_{p} y_{k}\right\|=0
$$

Also

$$
\begin{aligned}
\left\|P_{1} y_{k}\right\| & =\left\|S_{w_{k}}^{*} x_{1}\right\| /\left\|S_{w_{k}}^{*} x\right\| \geq\left\|P_{\mathcal{V}} S_{w_{k}}^{*} x_{1}\right\| /\left\|S_{w_{k}}^{*} x\right\| \\
& >\frac{\left\|x_{1}\right\|}{2\|x\|} \frac{\left\|P_{\mathcal{V}} S_{w_{k}}^{*} x\right\|}{\left\|S_{w_{k}}^{*} x\right\|}=\frac{\left\|x_{1}\right\|}{2\|x\|}\left\|P_{\mathcal{V}} y_{k}\right\| .
\end{aligned}
$$

By the compactness of the unit ball in $\mathcal{V}$, there is a subsequence of the $y_{k}$ 's which converges to a unit vector $v$ in $\mathcal{V}$. Clearly, $P_{p} v=0$, and thus $v$ belongs to $\mathcal{V}_{c} \cap \mathfrak{S}^{*}[x]$; whence this subspace is non-zero. By construction, $\left\|P_{1} v\right\| \geq\left\|x_{1}\right\| / 2\|x\|$, and therefore is also non-zero.

Corollary 4.2. Every non-zero subspace of $\mathcal{H}_{c}$ which is invariant for $\mathfrak{S}^{*}$ has non-zero intersection with $\mathcal{V}_{c}$. In particular $\mathcal{H}_{c}=\mathfrak{S}\left[\mathcal{V}_{c}\right]$.

Proof. Let $\mathcal{M}$ be any non-zero $\mathfrak{S}^{*}$-invariant subspace contained in $\mathcal{H}_{c}$. If $x$ is any non-zero vector in $\mathcal{M}$, the previous lemma applied to $x$ and $\mathcal{H}_{1}=\mathcal{H}_{c}$ shows that $\mathfrak{S}^{*}[x]$ intersects $\mathcal{V}_{c}$ non-trivially.

By Lemma 3.4, $\mathcal{N}=\mathfrak{S}\left[\mathcal{V}_{c}\right]$ reduces $\mathfrak{S}$. We claim that $\mathcal{N}=\mathcal{H}_{c}$. For otherwise, let $\mathcal{H}_{1}=\mathcal{H}_{c} \cap \mathcal{N}^{\perp}$. By the first paragraph, this reducing subspace for $\mathfrak{S}$ must intersect $\mathcal{V}_{c}$ non-trivially. So $\mathcal{H}_{1}$ is not orthogonal to $\mathcal{N}$, contrary to fact. Therefore $\mathcal{H}_{1}$ must be zero.

Corollary 4.3. Suppose that $\sum_{i=1}^{n} A_{i} A_{i}^{*}=I$ and $\mathfrak{A}=\mathcal{B}(\mathcal{V})$. Then every invariant subspace of $\mathfrak{S}^{*}$ contains $\mathcal{V}$.

Proof. Since $\mathcal{H}=\mathcal{H}_{c}$, any $\mathfrak{S}^{*}$-invariant subspace $\mathcal{M}$ intersects $\mathcal{V}$ in a non-trivial subspace. This subspace is invariant for $\left.\mathfrak{S}^{*}\right|_{\mathcal{V}}=\mathfrak{A}^{*}=\mathcal{B}(\mathcal{V})$. Hence it is all of $\mathcal{V}$.

Let $\mathfrak{B}$ denote the WOT-closed operator algebra on $\mathcal{H}=\mathcal{V} \oplus \mathcal{K}_{n}^{(\alpha)}$ spanned by $\mathcal{B}(\mathcal{H}) P_{\mathcal{V}}$ and $0_{\mathcal{V}} \oplus \mathfrak{L}_{n}^{(\alpha)}$.

Lemma 4.4. Every weak-* continuous functional on $\mathfrak{B}$ is given by a trace class operator of rank at most $d+1$. 
Proof. An element $B$ of $\mathfrak{B}$ is determined by $B P_{\mathcal{V}}$ and $B P_{\mathcal{V}}^{\perp}$. If $e_{1}, \ldots, e_{d}$ is a basis for $\mathcal{V}$, the former is determined by the vectors $B e_{j}$. The latter term is unitarily equivalent to $A^{(\alpha)}$ for some $A \in \mathfrak{L}_{n}$. Any functional $\varphi$ is thus determined by a functional $\varphi_{0}$ on $\mathfrak{L}_{n}^{(\alpha)}$ and by $d$ functionals on $\mathcal{H}$ given by the Riesz Representation Theorem by a vector $y_{j}$. By [15, Theorem 2.10], the functional on $\mathfrak{L}_{n}$ is given by a rank one functional $\varphi_{0}(A)=(A \eta, \zeta)$. Whence

$$
\varphi(B)=\sum_{j=1}^{d}\left(B e_{j}, y_{j}\right)+(B \eta, \zeta) .
$$

Corollary 4.5. The WOT and weak-* topologies coincide on $\mathfrak{B}$, and thus also on $\mathfrak{S}$. In particular, the weak-* closed algebra generated by the $S_{i}$ 's coincides with $\mathfrak{S}$.

\section{The Cuntz Case}

In this section, we specialize to the Cuntz case: $\sum_{i=1}^{n} A_{i} A_{i}^{*}=I$ for which the isometric dilation yields a representation of the Cuntz algebra.

Example 5.1. We begin with a description of the case in which $\mathcal{V}$ is one dimensional.. A special case of a finite correlated state is a Cuntz state. This is determined by scalars $\eta=\left(\eta_{1}, \ldots, \eta_{n}\right)$ such that $\sum_{i=1}^{n}\left|\eta_{i}\right|^{2}=1$. The state is determined by

$$
\varphi_{\eta}\left(s_{i_{1}} \ldots s_{i_{k}} s_{j_{1}}^{*} \ldots s_{j_{l}}^{*}\right)=\eta_{i_{1}} \ldots \eta_{i_{k}} \bar{\eta}_{j_{1}} \ldots \bar{\eta}_{j_{l}} .
$$

It is easy to show that the cyclic vector $\xi_{\eta}$ from the GNS construction $\left(\mathcal{H}_{\eta}, \pi_{\eta}, \xi_{\eta}\right)$ spans a one-dimensional space invariant for every $\pi_{\eta}\left(S_{i}^{*}\right)$. Indeed,

$$
\begin{aligned}
\left\|\pi_{\eta}\left(S_{i}^{*}\right) \xi_{\eta}-\bar{\eta}_{i} \xi_{\eta}\right\|^{2}= & \left\langle\pi_{\eta}\left(S_{i}^{*}\right) \xi_{\eta}, \pi_{\eta}\left(S_{i}^{*}\right) \xi_{\eta}\right\rangle-\eta_{i}\left\langle\pi_{\eta}\left(S_{i}^{*}\right) \xi_{\eta}, \xi_{\eta}\right\rangle \\
& -\bar{\eta}_{i}\left\langle\xi_{\eta}, \pi_{\eta}\left(S_{i}^{*}\right) \xi_{\eta}\right\rangle+\left|\eta_{i}\right|^{2} \\
= & \varphi_{\eta}\left(S_{i} S_{i}^{*}\right)-\left|\eta_{i}\right|^{2}=\left|\eta_{i}\right|^{2}-\left|\eta_{i}\right|^{2}=0 .
\end{aligned}
$$

The restrictions $A_{i}^{*}=\left.S_{i}^{*}\right|_{\operatorname{span}\left\{\xi_{\eta}\right\}}=\overline{\eta_{i}}$ satisfy $\sum_{i=1}^{n} A_{i} A_{i}^{*}=1$. They may be dilated to their minimal isometric dilation, which is necessarily the original $S_{i}$ since $\xi_{\eta}$ is a cyclic vector.

Specializing to the case of $\eta=(1,0, \ldots, 0)$, one has $A_{1}=1$ and $A_{i}=0$ for $2 \leq i \leq n$. This yields the atomic representation $\sigma_{1,1}$ mentioned in the Background section. In particular, the algebra $\mathfrak{S}$ is unitarily equivalent to $\mathfrak{B}_{n, 1}$. 
The various Cuntz states are related by the action of the gauge group $\mathcal{U}(n)$ which acts as an automorphism group on $\mathcal{O}_{n}$ and on the CuntzToeplitz algebra $\mathcal{E}_{n}$. Indeed, if we write Fock space $\mathcal{K}_{n}$ as a direct sum $\mathbb{C} \oplus \mathcal{H}_{n} \oplus \mathcal{H}_{n}^{\otimes 2} \oplus \mathcal{H}_{n}^{\otimes 3} \oplus \ldots$, where $\mathcal{H}_{n}$ is an $n$-dimensional Hilbert space, then each unitary matrix $U \in \mathcal{U}(n)$ determines a unitary operator $\widetilde{U}=I \oplus U \oplus U^{\otimes 2} \oplus U^{\otimes 3} \oplus \ldots$ on $\mathcal{K}_{n}$. Conjugation by $\widetilde{U}$ acts as an automorphism $\Theta_{U}$ of $\mathcal{E}_{n}$. Moreover, it maps the ideal of compact operators onto itself. So it also induces an automorphism $\theta_{U}$ of $\mathcal{O}_{n}$. If $U=\left[u_{i j}\right]$ is an $n \times n$ unitary matrix, this automorphism can also be seen to be given by

$$
\Theta_{U}\left(L_{j}\right)=\sum_{i=1}^{n} u_{i j} L_{i} \quad \text { for } \quad 1 \leq j \leq n .
$$

Given $\eta$, let $U$ be any unitary with $u_{1 j}=\eta_{j}$. Then it follows that

$$
\varphi_{\eta}(A)=\varphi_{(1,0, \ldots, 0)}\left(\theta_{U}(A)\right) \text { for all } A \in \mathcal{O}_{n} .
$$

So the corresponding representations are equivalent up to this automorphism. In particular, the algebras $\mathfrak{S}_{\eta}$ generated by these representations are unitarily equivalent even though the representations are not.

A crucial step in the analysis of atomic representations was to show that certain projections lie in the algebra $\mathfrak{S}$. Indeed, this is a major advantage of $\mathfrak{S}$ over the $\mathrm{C}^{*}$-algebra, which contains no non-trivial projections, and over the von Neumann algebra it generates, which contains too many. As a case in point, the projection $P_{\eta}=\xi_{\eta} \xi_{\eta}^{*}$ belongs to $\mathfrak{S}_{\eta}$. Indeed, it is the only non-trivial projection in the whole algebra $\mathfrak{S}_{\eta}$.

A crucial point of our analysis is the identification of projections in $\mathfrak{S}$ in greater generality. We begin with the irreducible case.

Theorem 5.2. Assume that $\sum_{i=1}^{n} A_{i} A_{i}^{*}=I$ and $\mathfrak{A}=\mathcal{B}(\mathcal{V})$. Then $\mathfrak{S}$ contains the projection $P_{\mathcal{V}}$.

Proof. Both $\mathfrak{S}$ and $P_{\mathcal{V}}$ belong to $\mathfrak{B}$. If $P_{\mathcal{V}}$ were not in $\mathfrak{S}$, Lemma 4.4 would provide aWOT-continuous functional $\varphi$ which annihilates $\mathfrak{S}$ such that $\varphi\left(P_{\mathcal{V}}\right)=1$. Represent $\varphi$ as a functional of rank $m$ in the form $\varphi(B)=\sum_{j=1}^{m}\left(B x_{j}, y_{j}\right)$. This then may be realized as a rank one functional on the $m$-fold ampliation of $\mathfrak{B}$. Indeed, form the vectors $x=\left(x_{1}, \ldots, x_{m}\right)$ and $y=\left(y_{1}, \ldots, y_{m}\right)$. Then $\varphi(B)=\left(B^{(m)} x, y\right)$.

Now the fact that $\varphi$ annihilates $\mathfrak{S}$ means that $x$ is orthogonal to the subspace $\mathcal{M}=\mathfrak{S}^{*(m)}[y]$. The algebra $\mathfrak{S}^{(m)}$ is generated by isometries $S_{i}^{(m)}$, which form the minimal dilation of the $A_{i}^{(m)}$ 's. So Corollary 4.2 of 
the Key Technical Lemma applies, and shows that $\mathcal{M}$ intersects $\mathcal{V}^{(m)}$ in a non-zero subspace $\mathcal{M}_{0}$ which is invariant for $\mathfrak{S}^{*(m)}$, and thus for $\mathfrak{A}^{*(m)}$.

By hypothesis, $\mathfrak{A}^{*(m)}=\mathcal{B}(\mathcal{V})^{(m)} \simeq \mathcal{B}(\mathcal{V}) \otimes \mathbb{C}^{m}$, which is a finite dimensional $\mathrm{C}^{*}$-algebra. The invariant subspace $\mathcal{M}_{0}$ is thus the range of a projection $Q$ in the commutant $\mathbb{C}^{d} \otimes \mathfrak{M}_{m}$. Let $\tilde{Q}$ denote the operator in $\mathbb{C} I_{\mathcal{H}} \otimes \mathfrak{M}_{m}$ acting on $\mathcal{H}^{(m)}$ with the same matrix coefficients as $Q$. That is, $\tilde{Q}$ is the unique operator in $\left(\mathcal{B}(\mathcal{H}) \otimes \mathbb{C}^{m}\right)^{\prime}$ such that $P_{\mathcal{V}}^{(m)} \tilde{Q}=Q$.

The projection $\tilde{Q}$ yields a decomposition of $\mathcal{H}^{(m)}$ into S-reducing subspaces $\mathcal{H}_{1} \oplus \mathcal{H}_{2}$ where $\mathcal{H}_{1}=\operatorname{ker} \tilde{Q}$ and $\mathcal{H}_{2}=\operatorname{Ran} \tilde{Q}$; and likewise $\mathcal{V}^{(m)}=\mathcal{V}_{1} \oplus \mathcal{V}_{2}$ where

$$
\mathcal{V}_{1}:=\mathcal{H}_{1} \cap \mathcal{V}^{(m)}=\operatorname{ker} Q \quad \text { and } \quad \mathcal{V}_{2}:=\mathcal{H}_{2} \cap \mathcal{V}^{(m)}=\operatorname{Ran} Q
$$

Observe that $\mathcal{M}_{0}$ is contained in $\mathcal{H}_{2}$. For if we had a vector $x \in \mathcal{M}$ such that $P_{\mathcal{H}_{1}} x \neq 0$, then our Key Lemma 4.1 implies that there is a non-zero vector $v$ in $\mathcal{M} \cap \mathcal{V}^{(m)}=\mathcal{M}_{0}$ such that $P_{\mathcal{H}_{1}} v \neq 0$. But by definition of $Q$ and $\widetilde{Q}, \mathcal{M}_{0}$ is orthogonal to $\mathcal{H}_{1}$, a contradiction.

In particular, as $y \in \mathcal{M}$, we have $y=\tilde{Q} y$. Thus

$$
P_{\mathcal{V}}^{(m)} y=P_{\mathcal{V}}^{(m)} \tilde{Q} y=Q P_{\mathcal{V}}^{(m)} y
$$

belongs to $Q \mathcal{V}^{(m)}=\mathcal{M}_{0}$. Since $x$ is orthogonal to $\mathcal{M}$ and hence to $\mathcal{M}_{0}$, we see that

$$
\varphi\left(P_{\mathcal{V}}\right)=\left(P_{\mathcal{V}}^{(m)} x, y\right)=\left(x, P_{\mathcal{V}}^{(m)} y\right)=0 .
$$

Consequently $P_{\mathcal{V}}$ belongs to $\mathfrak{S}$.

This immediately yields a structure theorem for $\mathfrak{S}$. Note that this does not classify the associated representations, as they depend on the specific generators, not just the algebra.

Corollary 5.3. Assume that $\sum_{i=1}^{n} A_{i} A_{i}^{*}=I$ and $\mathfrak{A}=\mathcal{B}(\mathcal{V})$. Then $\mathfrak{S} \simeq \mathcal{B}(\mathcal{H}) P_{\mathcal{V}}+\left(0_{\mathcal{V}} \oplus \mathfrak{L}_{n}^{((n-1) d)}\right) \simeq \mathfrak{B}_{n, d}$

Proof. By Theorem 5.2, $\mathfrak{S}$ contains $P_{\mathcal{V}}$. Therefore it contains $P_{\mathcal{V}} \mathfrak{S}=$ $\mathcal{B}(\mathcal{V})$. Moreover, it contains $S_{i} P_{\mathcal{V}}^{\perp} \simeq 0_{\mathcal{V}} \oplus L_{i}^{(\alpha)}$, where $\alpha=(n-1) d$. Thus $\mathfrak{S}$ contains the WOT-closed algebra that these operators generate, which is evidently $0_{\mathcal{V}} \oplus \mathfrak{L}_{n}^{(\alpha)}$. Finally, if $v$ is any non-zero vector in $\mathcal{V}$, $\mathfrak{S}[v]$ contains $\mathcal{V}$ by hypothesis. So it is all of $\mathcal{H}$ by minimality of the dilation. Therefore for any $x \in \mathcal{H}$, there are operators $T_{k} \in \mathfrak{S}$ such that $T_{k} v$ converges to $x$. Thus $\mathfrak{S}$ contains $T_{k} v v^{*}$, which converge to the rank one operator $x v^{*}$. So $\mathcal{B}(\mathcal{H}) P_{\mathcal{V}}$ belongs to $\mathfrak{S}$. This is the whole 
WOT-closed algebra which we called $\mathfrak{B}$, which trivially contains $\mathfrak{S}$. It is evident that $\mathfrak{B}$ is unitarily equivalent to $\mathfrak{B}_{n, d}$.

Now suppose that $\mathfrak{A}$ is a more general subalgebra of $\mathcal{B}(\mathcal{V})$. We wish to determine the structure of $\mathfrak{S}$ from information about $\mathfrak{A}$.

Lemma 5.4. Assume that $\sum_{i=1}^{n} A_{i} A_{i}^{*}=I$. Suppose that $\mathcal{V}$ contains a minimal $\mathfrak{A}^{*}$-invariant subspace $\mathcal{V}_{0}$ of dimension $d_{0}$ which is cyclic for $\mathfrak{A}$. Then $\mathfrak{S}$ contains $\mathcal{B}(\mathcal{H}) P_{\mathcal{V}_{0}}$, and is unitarily equivalent to $\mathfrak{B}_{n, d_{0}}$.

Proof. By Burnside's Theorem [32, Corollary 8.6], since $\left.\mathfrak{A}^{*}\right|_{\mathcal{V}_{0}}$ has no proper invariant subspaces, it must equal all of $\mathcal{B}\left(\mathcal{V}_{0}\right)$. Let $\mathcal{H}_{0}=\mathfrak{S}\left[\mathcal{V}_{0}\right]$. This is a reducing subspace for $\mathfrak{S}$ by Lemma 3.4. We will argue that $\mathcal{H}_{0}=\mathcal{H}$.

Suppose that $x$ is a non-zero vector orthogonal to $\mathcal{H}_{0}$. By Corollary 4.2 of the Key Technical Lemma, $\mathfrak{S}^{*}[x] \cap \mathcal{V}$ contains a non-zero vector $v$. Moreover since $\mathcal{H}_{0}^{\perp}$ reduces $\mathfrak{S}, v$ is orthogonal to $\mathcal{H}_{0}$. Therefore $\mathfrak{S}^{*}[v]=\mathfrak{A}^{*}[v]$ is an $\mathfrak{A}^{*}$-invariant subspace orthogonal to $\mathcal{V}_{0}$. Since $\mathfrak{A} \mathcal{V}_{0}=\mathcal{V}$, there is an $A \in \mathfrak{A}$ and $v_{0} \in \mathcal{V}_{0}$ such that $A v_{0}=v$. So that

$$
\|v\|^{2}=\left(A v_{0}, v\right)=\left(v_{0}, A^{*} v\right)=0 .
$$

This contradiction establishes our claim.

Now consider the compressions $\widetilde{A}_{i}=P_{\mathcal{V}_{0}} A_{i} \mid \mathcal{V}_{0}=\left(\left.A_{i}^{*}\right|_{\mathcal{V}_{0}}\right)^{*}$. Then $\sum_{i=1}^{n} \widetilde{A}_{i} \widetilde{A}_{i}^{*}=I_{\mathcal{V}_{0}}$ follows from the $\mathfrak{A}^{*}$-invariance of $\mathcal{V}_{0}$. Also by hypothesis, the algebra $\widetilde{\mathfrak{A}}$ generated by the $\widetilde{A}_{i}$ 's is $\mathcal{B}\left(\mathcal{V}_{0}\right)$. The minimal dilation of this $n$-tuple must be precisely the restriction of $S_{i}$ to $\mathfrak{S}\left[\mathcal{V}_{0}\right]=\mathcal{H}$, which is $S_{i}$. So by Corollary 5.3, it follows that $\mathfrak{S}$ is unitarily equivalent to $\mathfrak{B}_{n, d_{0}}$.

The following corollary is almost immediate from the structure of $\mathfrak{B}_{n, d_{0}}$. We point it out in order to obtain some non-trivial consequences.

Corollary 5.5. Assume that $\sum_{i=1}^{n} A_{i} A_{i}^{*}=I$. If $\mathcal{V}$ contains a subspace $\mathcal{V}_{0}$ which is cyclic for $\mathfrak{A}$ and is a minimal invariant subspace for $\mathfrak{A}^{*}$, then $\mathcal{V}_{0}$ is the unique minimal $\mathfrak{A}^{*}$-invariant subspace.

Proof. We have $\mathfrak{A}^{*}=\left.\mathfrak{S}^{*}\right|_{\mathcal{V}}$. So by the previous lemma, $\mathfrak{A}^{*}$ contains $P_{\mathcal{V}_{0}} \mathcal{B}(\mathcal{V})$. Consequently, $\mathcal{V}_{0}$ is contained in every non-zero $\mathfrak{A}^{*}$-invariant subspace.

Remark 5.6. This puts constraints on which subalgebras $\mathfrak{A}$ of $\mathcal{B}(\mathcal{V})$ can be generated by $A_{i}$ 's which satisfy $\sum_{i=1}^{n} A_{i} A_{i}^{*}=I$. For example, the semisimple algebra of matrices of the form $\mathfrak{A}_{t}=\left[\begin{array}{cc}a & 0 \\ (b-a) t & b\end{array}\right]$ for $a, b$ in $\mathbb{C}$ and a fixed $t \neq 0$ is similar to the $2 \times 2$ diagonal algebra. Note that $\mathfrak{A}_{t}$ has two independent vectors which are cyclic for $\mathfrak{A}_{t}$ and eigenvalues for 
$\mathfrak{A}_{t}^{*}$, namely $e_{1}$ and $f_{2}=-\bar{t} e_{1}+e_{2}$. By the corollary above, this cannot equal the algebra $\mathfrak{A}$. Indeed, if the generators of our algebra were $A_{i}=\left[\begin{array}{cc}a_{i} & 0 \\ \left(b_{i}-a_{i}\right) t & b_{i}\end{array}\right]$, then a computation would show that $\sum_{i=1}^{n}\left|a_{i}\right|^{2}=1$. Likewise considering the matrix with respect to an orthonormal basis $\left\{f_{1}, f_{2}\right\}$ would show that $\sum_{i=1}^{n}\left|b_{i}\right|^{2}=1$. This then forces $\sum_{i=1}^{n} \mid a_{i}-$ $\left.b_{i}\right|^{2}|t|^{2}=0$. Since $t \neq 0$, this forces all the $A_{i}$ 's to be scalar, and hence they do not generate $\mathfrak{A}_{t}$.

Example 5.7. Consider a special case of the previous corollary: if $\mathfrak{A}$ has a cyclic vector $e$ which is an eigenvalue for $\mathfrak{A}^{*}$. Then $\mathfrak{S}$ is unitarily equivalent to $\mathfrak{B}_{n, 1}$. The algebra $\mathfrak{A}$ decomposes as $\mathfrak{A}=\mathcal{B}(\mathcal{V}) P_{e}+J \mathfrak{A}_{1} P_{e}^{\perp}$ where $P_{e}$ is the orthogonal projection onto $\mathbb{C} e, J$ is the injection of $\mathcal{V}_{1}=\{e\}^{\perp}$ into $\mathcal{V}$, and $\mathfrak{A}_{1}$ is a unital subalgebra of $\mathcal{B}\left(\mathcal{V}_{1}\right)$. It is easy to see that

$$
\text { Lat } \mathfrak{A}=\left\{\mathcal{V}, J M: M \in \operatorname{Lat} \mathfrak{A}_{1}\right\} .
$$

Hence if $\mathfrak{B}_{1}=\operatorname{Alg}$ Lat $\mathfrak{A}_{1}$, then

$$
\mathfrak{B}:=\operatorname{Alg} \text { Lat } \mathfrak{A}=\mathcal{B}(\mathcal{V}) P_{e}+J \mathfrak{B}_{1} P_{e}^{\perp} .
$$

It follows that $\mathfrak{A}$ is reflexive if and only if $\mathfrak{A}_{1}$ is.

Thus if $\operatorname{dim} \mathcal{V}_{1}>1$, there are non-reflexive examples. For example, consider the non-reflexive algebra $\mathfrak{A}_{1}=\left\{\left[\begin{array}{ll}a & 0 \\ b & a\end{array}\right]: a, b \in \mathbb{C}\right\}$. Take $n=3$ and let

$$
A_{1}=\left[\begin{array}{ccc}
1 & 0 & 0 \\
0 & 1 / \sqrt{2} & 0 \\
0 & 0 & 1 / \sqrt{2}
\end{array}\right] \quad A_{2}=\left[\begin{array}{ccc}
0 & 0 & 0 \\
0 & 0 & 0 \\
1 / 2 & 1 / 2 & 0
\end{array}\right] \quad A_{3}=\left[\begin{array}{ccc}
0 & 0 & 0 \\
1 / \sqrt{2} & 0 & 0 \\
0 & 0 & 0
\end{array}\right]
$$

This can be seen to satisfy $\sum_{i=1}^{3} A_{i} A_{i}^{*}=I_{3}$ and to generate the algebra $\mathfrak{A}=\left\{\left[\begin{array}{lll}c & 0 & 0 \\ d & a & 0 \\ e & b & a\end{array}\right]: a, b, c, d, e \in \mathbb{C}\right\}$. This is not reflexive.

Nevertheless, $\mathfrak{A}^{*}$ has a unique minimal invariant subspace, and thus $\mathfrak{S}$ is unitarily equivalent to $\mathfrak{B}_{3,1}$, which is hyper-reflexive. So there is no direct correspondence between the reflexivity of $\mathfrak{A}$ and $\mathfrak{S}$.

Lemma 5.8. Let $A=\left(A_{1}, \ldots, A_{n}\right)$ be an n-tuple on a finite dimensional space $\mathcal{V}$ such that $\sum_{i=1}^{n} A_{i} A_{i}^{*}=I$. Let $\mathfrak{A}$ be the unital algebra that they generate. Let $S=\left(S_{1}, \ldots, S_{n}\right)$ be the minimal isometric dilation, and $\mathfrak{S}$ the WOT-closed algebra they generate. Then $\mathfrak{S}$ is irreducible if and only if $\mathfrak{A}^{*}$ has a unique minimal invariant subspace $\mathcal{V}_{0}$.

Proof. If $\mathcal{V}_{0}$ is unique, then it must be cyclic for $\mathfrak{A}$ since $\mathcal{V} \ominus \mathfrak{A}\left[\mathcal{V}_{0}\right]$ is an invariant subspace of $\mathfrak{A}^{*}$ orthogonal to $\mathcal{V}_{0}$. So Lemma 5.4 applies. Since $\mathfrak{S}$ contains $\mathcal{B}(\mathcal{H}) P_{\mathcal{V}_{0}}$, it is evidently irreducible. 
Indeed, this conclusion follows if there is any minimal $\mathfrak{A}^{*}$-invariant subspace $\mathcal{V}_{0}$ which is cyclic for $\mathfrak{A}$. By Corollary 5.5, $\mathcal{V}_{0}$ is necessarily the unique minimal $\mathfrak{A}^{*}$-invariant subspace.

Finally suppose that there is a minimal $\mathfrak{A}^{*}$-invariant subspace $\mathcal{V}_{0}$ which is not cyclic. Then as in the first paragraph, $\mathcal{V} \ominus \mathfrak{A}\left[\mathcal{V}_{0}\right]$ is an invariant subspace of $\mathfrak{A}^{*}$ orthogonal to $\mathcal{V}_{0}$. Let $\mathcal{V}_{1}$ be a minimal $\mathfrak{A}^{*}$ invariant subspace contained therein. Notice that $\mathfrak{S}\left[\mathcal{V}_{i}\right]$ are pairwise orthogonal reducing subspaces for $\mathfrak{S}$ by Lemma 3.4. Hence $\mathcal{H}$ contains proper reducing subspaces, and so $\mathfrak{S}$ is reducible.

Now we see how to deal with the case of more than one minimal $\mathfrak{A}^{*}$ invariant subspace. In this lemma, we do not concern ourselves with questions of uniqueness.

Lemma 5.9. Assume that $\sum_{i=1}^{n} A_{i} A_{i}^{*}=I$. There is a family of minimal $\mathfrak{A}^{*}$-invariant subspaces $\mathcal{V}_{j}$ of $\mathcal{V}, 1 \leq j \leq s$, such that $\mathcal{H}$ decomposes into an orthogonal direct sum of $\mathcal{H}_{j}=\mathfrak{S}\left[\mathcal{V}_{j}\right]$; and the algebras $\left.\mathfrak{S}\right|_{\mathcal{H}_{j}}$ are irreducible.

Proof. This is just a matter of choosing a maximal family of pairwise orthogonal minimal $\mathfrak{A}^{*}$-invariant subspaces, say $\mathcal{V}_{j}$ for $1 \leq j \leq s$. By Lemma 3.4, the subspaces $\mathcal{H}_{j}=\mathfrak{S}\left[\mathcal{V}_{j}\right]$ are pairwise orthogonal and reducing for $\mathfrak{S}$. Moreover a direct application of the previous lemma applied to $\mathcal{H}_{j}$ and $\mathcal{V}_{j}$ shows that $\left.\mathfrak{S}\right|_{\mathcal{H}_{j}}$ is irreducible. Finally we must show that $\sum_{j=1}^{\oplus s} \mathcal{H}_{j}=\mathcal{H}$. Take any vector $x$ orthogonal to this sum. By Corollary 4.2 of the Key Technical Lemma, $\mathfrak{S}^{*}[x]$ intersects $\mathcal{V}$ in a non-zero $\mathfrak{A}^{*}$-invariant subspace orthogonal to all of the $\mathcal{H}_{j}$ 's, and thus orthogonal to all of the $\mathcal{V}_{j}$ 's. This is contrary to construction, and so yields a contradiction.

Given an $n$-tuple $A=\left(A_{1}, \ldots, A_{n}\right)$ such that $\sum_{i=1}^{n} A_{i} A_{i}^{*}=I$, let us pick a maximal family of mutually orthogonal minimal $\mathfrak{A}^{*}$-invariant subspaces $\mathcal{V}_{j}$ of $\mathcal{V}, 1 \leq j \leq s$; and let $P_{j}=P_{\mathcal{V}_{j}}$. From the minimality of each $\mathcal{V}_{j}$ as an $\mathfrak{A}^{*}$-invariant subspace, we know that $P_{j} \mathfrak{A}^{*} P_{j}=\mathcal{B}\left(\mathcal{V}_{j}\right)$. Set $\widetilde{\mathcal{V}}=\sum_{j=1}^{\oplus s} \mathcal{V}_{j}$. Let $\widetilde{A}_{i}=\left.P_{\widetilde{\mathcal{V}}} A_{i}\right|_{\tilde{\mathcal{V}}}=\left(\left.A_{i}^{*}\right|_{\tilde{\mathcal{V}}}\right)^{*}$ be the compression of $A_{i}$ to $\widetilde{\mathcal{V}}$; and let $\widetilde{\mathfrak{A}}$ denote the algebra they generate in $\mathcal{B}(\widetilde{\mathcal{V}})$.

Notice that the minimal isometric dilation of $\widetilde{A}=\left(\widetilde{A}_{1}, \ldots, \widetilde{A}_{n}\right)$ is precisely $S$. It is evident that $S$ is a joint isometric dilation of $\widetilde{A}$. To show that it is minimal, it suffices to show that $\mathfrak{S}[\widetilde{\mathcal{V}}]=\mathcal{H}$. But this is established above in Lemma 5.9.

Our goal is to show that $\widetilde{\mathfrak{A}}$ is a $\mathrm{C}^{*}$-algebra. For the moment, let us show that it is semisimple. Note that $\widetilde{\mathfrak{A}}$ is contained in $\sum_{1 \leq j \leq s}^{\oplus} \mathcal{B}\left(\mathcal{V}_{j}\right)$. Moreover the quotient map $q_{j}$ of compression to $\mathcal{V}_{j}$ maps $\mathfrak{A}$ onto $\mathcal{B}\left(\mathcal{V}_{j}\right)$. 
Thus the kernel of this map is a maximal ideal. Since $\sum^{\oplus} q_{j}=$ id is faithful, the intersection of all maximal ideals is $\{0\}$. Hence $\widetilde{\mathfrak{A}}$ is semisimple.

Indeed, there is a minimal family $G$ so that $\sum_{g \in G}^{\oplus} q_{g}$ is faithful. By the Wedderburn theory, the minimal ideal $\mathfrak{A}_{g}=\operatorname{ker} \sum_{h \in G \backslash\{g\}}^{\oplus} q_{h}$ is isomorphic to $\mathcal{B}\left(\mathcal{V}_{g}\right)$. But this kernel will, in practice, be supported on several of the $\mathcal{V}_{j}$ 's. This yields a partition $\widetilde{\mathcal{V}}=\sum_{g \in G}^{\oplus} \mathcal{W}_{g}$ where $\mathcal{W}_{g}=\sum_{j \in G_{g}}^{\oplus} \mathcal{V}_{j}$ is a sum of those $\mathcal{V}_{j}$ 's equivalent to $\mathcal{V}_{g}$. Because $\mathcal{B}\left(\mathcal{V}_{g}\right)$ is simple, it follows that there is an algebra isomorphism $\sigma_{j}$ of $\mathcal{B}\left(\mathcal{V}_{g}\right)$ onto $\mathcal{B}\left(\mathcal{V}_{j}\right)$ for each $j \in G_{g}$ such that

$$
\left.\widetilde{\mathfrak{A}}\right|_{\mathcal{W}_{g}} \simeq\left\{\sum_{j \in G_{g}}^{\oplus} \sigma_{j}(X): X \in \mathcal{B}\left(\mathcal{V}_{g}\right)\right\} .
$$

It is well-known that every isomorphism between $\mathcal{B}\left(\mathcal{V}_{g}\right)$ and $\mathcal{B}\left(\mathcal{V}_{j}\right)$ is spatial: $\sigma_{j}(X)=T_{j} X T_{j}^{-1}$ for some invertible operator $T_{j}$, which is unique up to a scalar multiple.

We also need to consider the unital completely positive map $\Phi$ on $\mathcal{B}(\widetilde{\mathcal{V}})$ given by

$$
\Phi(X)=\sum_{i=1}^{n} \widetilde{A}_{i} X \widetilde{A}_{i}^{*}
$$

Suppose that two blocks $\mathcal{V}_{1}$ and $\mathcal{V}_{2}$ are related by a similarity as above. Let $B_{i}:=\left.P_{\mathcal{V}_{1}} A_{i}\right|_{\mathcal{V}_{1}}$ and $C_{i}:=\left.P_{\mathcal{V}_{2}} A_{i}\right|_{\mathcal{V}_{2}}=T B_{i} T^{-1}$. Since

$$
\sum_{i=1}^{n} B_{i} B_{i}^{*}=I_{\mathcal{V}_{1}} \quad \text { and } \quad \sum_{i=1}^{n} C_{i} C_{i}^{*}=I_{\mathcal{V}_{2}},
$$

we compute that

$$
I_{\mathcal{V}_{2}}=\sum_{i=1}^{n}\left(T B_{i} T^{-1}\right)\left(T B_{i} T^{-1}\right)^{*}=T \Phi_{1}\left(T^{-1} T^{*-1}\right) T^{*},
$$

where $\Phi_{1}(X)=\sum_{i=1}^{n} B_{i} X B_{i}^{*}=\left.P_{1} \Phi\left(P_{1} X P_{1}\right)\right|_{\mathcal{V}_{1}}$. Therefore

$$
\Phi_{1}\left(T^{-1} T^{*-1}\right)=T^{-1} T^{*-1} .
$$

We now study this completely positive map in order to gain information about the structure of $\widetilde{\mathfrak{A}}$.

Lemma 5.10. Let $\Phi(X)=\sum_{i=1}^{n} A_{i} X A_{i}^{*}$ be a unital completely positive map on $\mathcal{B}(\mathcal{V})$, where $\mathcal{V}$ is finite dimensional. If there is a non-scalar operator $X$ such that $\Phi(X)=X$, then $\mathfrak{A}^{*}=\operatorname{Alg}\left\{A_{1}^{*}, \ldots, A_{n}^{*}\right\}$ has two pairwise orthogonal minimal invariant subspaces. 
Proof. Since $\Phi$ is self-adjoint and unital, there is a positive non-scalar $X$ such that $\Phi(X)=X$. Let $\|X\|=1$ and let $\mu$ denote the smallest eigenvalue of $X$. Then $\mathcal{M}=\operatorname{ker}(X-I)$ and $\mathcal{N}=\operatorname{ker}(X-\mu I)$ are pairwise orthogonal non-zero subspaces. For any unit vector $x \in \mathcal{M}$,

$$
\begin{aligned}
\|x\|^{2} & =(\Phi(X) x, x)=\sum_{i=1}^{n}\left(X A_{i}^{*} x, A_{i}^{*} x\right) \\
& \leq \sum_{i=1}^{n}\left(A_{i}^{*} x, A_{i}^{*} x\right)=\|x\|^{2}
\end{aligned}
$$

This equality can only hold if each $A_{i}^{*} x$ belongs to $\mathcal{M}$. Hence $\mathcal{M}$ is invariant for $\mathfrak{A}^{*}$.

This argument worked because 1 is an extreme point in the spectrum of $X$. This is also the case for $\mu$. Hence a similar argument shows that $\mathcal{N}$ is invariant for $\mathfrak{A}^{*}$.

The following is a partial converse to the previous lemma.

Lemma 5.11. Let $\Phi(X)=\sum_{i=1}^{n} A_{i} X A_{i}^{*}$ be a unital completely positive map on $\mathcal{B}(\mathcal{V})$, where $\mathcal{V}$ is finite dimensional. Suppose that $A_{i}=B_{i} \oplus C_{i}$ with respect to an orthogonal decomposition $\mathcal{V}=\mathcal{V}_{1} \oplus \mathcal{V}_{2}$. Moreover, suppose that $\operatorname{Alg}\left\{B_{i}\right\}=\mathcal{B}\left(\mathcal{V}_{1}\right)$ and $\operatorname{Alg}\left\{C_{i}\right\}=\mathcal{B}\left(\mathcal{V}_{2}\right)$. If there is an operator $X$ such that $\Phi(X)=X$ and $X_{21}:=P_{\mathcal{V}_{2}} X P_{\mathcal{V}_{1}} \neq 0$, then there is a unitary operator $W$ such that $C_{i}=W^{*} B_{i} W$. Moreover the fixed point set of $\Phi$ consists of all matrices of the form $\left[\begin{array}{cc}a_{11} I_{\mathcal{V}_{1}} & a_{12} W^{*} \\ a_{21} W & a_{22} I_{\mathcal{V}_{2}}\end{array}\right]$.

Proof. Since $\Phi$ is self-adjoint, we may suppose that $X=X^{*}$. Then normalize so that $\left\|X_{21}\right\|=1$. Let $\mathcal{M}=\left\{v \in \mathcal{V}_{1}:\left\|X_{21} v\right\|=\|v\|\right\}$. Also let $\mathcal{N}=X_{21} \mathcal{M}$ denote the corresponding subspace of $\mathcal{V}_{2}$. Write $B=\left[\begin{array}{lll}B_{1} & \ldots & B_{n}\end{array}\right]$ and $C=\left[\begin{array}{lll}C_{1} & \ldots & C_{n}\end{array}\right]$, so that

$$
X_{21} v=\Phi\left(X_{21}\right) v=C X_{21}^{(n)} B^{*} v \quad \text { for } \quad v \in \mathcal{M} .
$$

Since $C$ and $B^{*}$ are contractions, and $X_{21}$ achieves its norm on $v$, it follows that $B^{*} v$ belongs to the subspace $\mathcal{M}^{(n)}$ on which $X_{21}^{(n)}$ achieves its norm. Consequently each $B_{i}^{*}$ leaves $\mathcal{M}$ invariant. But as $\operatorname{Alg}\left\{B_{i}\right\}=$ $\mathcal{B}\left(\mathcal{V}_{1}\right)$, this forces $\mathcal{M}=\mathcal{V}_{1}$. Similarly, consideration of $X_{12}=X_{21}^{*}$ shows that $\mathcal{N}=\mathcal{V}_{2}$. Thus $X_{21}$ and $X_{21}^{*}$ are isometries; so $W=X_{21} \mid \mathcal{V}_{1}$ is a unitary map from $\mathcal{V}_{1}$ onto $\mathcal{V}_{2}$.

Further, the identity above now shows that $W=C W^{(n)} B^{*}$. Hence for all $v \in \mathcal{V}_{1}$

$$
\|v\|=\|W v\|=\left\|C W^{(n)} B^{*} v\right\| \leq\left\|W^{(n)} B^{*} v\right\| \leq\|v\|
$$


In particular, $C$ acts as an isometry from the range of $W^{(n)} B^{*}$ onto the range $\operatorname{Ran} W=\mathcal{V}_{2}$. Since $C$ is contractive, it must be zero on the orthogonal complement of $\operatorname{Ran} W^{(n)} B^{*}$. This implies that $C^{*}$ is an isometry of $\mathcal{V}_{2}$ onto $\operatorname{Ran} W^{(n)} B^{*}$. Consequently, $C^{*} W=W^{(n)} B^{*}$; or equivalently, $C_{i}^{*}=W B_{i}^{*} W^{*}$ for $1 \leq i \leq n$.

Finally, if $Y \in \mathcal{B}\left(\mathcal{V}_{1}, \mathcal{V}_{2}\right)$ and $\left[\begin{array}{ll}0 & 0 \\ Y & 0\end{array}\right]$ is fixed by $\Phi$, then

$$
Y=\sum_{i=1}^{n} C_{i} Y B_{i}^{*}=\sum_{i=1}^{n} W B_{i} W^{*} Y B_{i}^{*}=W \Phi_{1}\left(W^{*} Y\right)
$$

where $\Phi_{1}(X)=\sum_{i=1}^{n} B_{i} X B_{i}^{*}$ acts on $\mathcal{B}\left(\mathcal{V}_{1}\right)$. By Lemma $5.10, W^{*} Y$ is scalar; so $Y$ is a multiple of $W$. A similar analysis works for the other coordinates.

Example 5.12. Let

$$
A_{1}=\left[\begin{array}{ccc}
1 / \sqrt{2} & 0 & 0 \\
1 / 2 \sqrt{2} & 1 / 2 & 1 / 2 \sqrt{2} \\
0 & 0 & 1 / \sqrt{2}
\end{array}\right] \text { and } A_{2}=\left[\begin{array}{ccc}
1 / \sqrt{2} & 0 & 0 \\
-1 / 2 \sqrt{2} & 1 / 2 & -1 / 2 \sqrt{2} \\
0 & 0 & 1 / \sqrt{2}
\end{array}\right]
$$

Then the matrix $X=\left[\begin{array}{ccc}1 & 0 & 0 \\ 0 & 1 / 2 & 0 \\ 0 & 0 & 0\end{array}\right]$ satisfies $\Phi(X)=X$. A calculation shows that the fixed point set of $\Phi$ is the set of matrices $X=\left[x_{i j}\right]$ such that $x_{12}=x_{21}=x_{23}=x_{32}=0$ and $x_{11}+x_{13}+x_{31}+x_{33}=2 x_{22}$. In particular, this is not an algebra. The algebra $\mathfrak{A}^{*}$ has two minimal invariant subspaces, $\mathbb{C} e_{1}$ and $\mathbb{C} e_{3}$. Note that the compression of $\mathfrak{A}$ to $\operatorname{span}\left\{e_{1}, e_{3}\right\}$ consists of scalar matrices, and the fixed point set of the restricted completely positive map is the full $2 \times 2$ matrix algebra.

We can now utilize the detailed information about the map $\Phi$ to determine the algebra $\widetilde{\mathfrak{A}}$.

Theorem 5.13. Let $\Phi(X)=\sum_{i=1}^{n} A_{i} X A_{i}^{*}$ be a unital completely positive map on $\mathcal{B}(\mathcal{V})$, where $\mathcal{V}$ is finite dimensional. Suppose that $\mathcal{V}$ is the orthogonal direct sum of minimal $\mathfrak{A}^{*}$-invariant subspaces. Then $\mathfrak{A}$ is a $C^{*}$-algebra and the fixed point set of $\Phi$ coincides with the commutant of $\mathfrak{A}$.

Proof. Let $\mathcal{V}=\sum_{j}^{\oplus} \mathcal{V}_{j}$ be an orthogonal decomposition into minimal $\mathfrak{A}^{*}$-invariant subspaces. The restriction of $\mathfrak{A}$ to $\mathcal{V}_{j}$ is all of $\mathcal{B}\left(\mathcal{V}_{j}\right)$ by Burnside's Theorem. Thus the restriction of $\Phi$ to $\mathcal{B}\left(\mathcal{V}_{j}\right)$ maps onto the scalars by Lemma 5.10. By the earlier analysis, $\mathfrak{A}$ splits into an algebraic direct sum of minimal ideals which are isomorphic to full matrix algebras. These are determined by certain spatial intertwining relations between some of the summands. If the restriction of $A_{i}$ 's to $\mathcal{V}_{1}$ and $\mathcal{V}_{2}$ are related by an intertwining operator $T$, then we showed 
that $\Phi_{1}\left(T^{-1} T^{*-1}\right)=T^{-1} T^{*-1}$. But this is scalar by Lemma 5.10. So after scaling $T$, it becomes a unitary. It follows that $\mathfrak{A}$ is a $\mathrm{C}^{*}$-algebra.

Evidently, $\Phi$ fixes the commutant of $\mathfrak{A}=\mathfrak{A}^{*}$. Suppose that $\Phi(X)=$ $X$. If $\mathcal{V}_{k}$ and $\mathcal{V}_{l}$ are not related by a unitary intertwining map, then by Lemma 5.11, $P_{k} X P_{l}=0$. While if they are related by a unitary $W_{k l}$, then $P_{k} X P_{l}=x_{k l} W_{k l}$ belongs to $\mathfrak{A}^{\prime}$. It follows that the fixed point set is precisely the commutant of $\mathfrak{A}$.

Now it is possible to provide a complete description of the algebra $\mathfrak{S}$ in the Cuntz case.

Lemma 5.14. Let $P_{g}$ for $g \in G$ denote the minimal central projections in $\widetilde{\mathfrak{A}}$. These projections belong to $\mathfrak{S}$.

Proof. We follow the lines of Theorem 5.2. We may work in the algebra $\mathfrak{B}=\mathcal{B}(\mathcal{H}) P_{\mathcal{V}}+\left(0_{\mathcal{V}} \oplus \mathfrak{L}_{n}^{(\alpha)}\right)$ which contains $\mathfrak{S}$ and each projection $P_{g}$. If a central projection $P$ of $\widetilde{\mathfrak{A}}$ were not in $\mathfrak{S}$, by Lemma 4.4 it could be separated from $\mathfrak{S}$ by a functional of rank $d+1$, which as before we write as $\varphi(A)=\left(A^{(d+1)} x, y\right)$. Let $\mathcal{M}=\mathfrak{S}^{*(d+1)}[y]$ and $\mathcal{M}_{0}=\widetilde{\mathcal{V}}^{(d+1)} \cap \mathcal{M}$. This subspace $\mathcal{M}_{0}$ is invariant for the $C^{*}$-algebra $\widetilde{\mathfrak{A}}^{*(d+1)}=\widetilde{\mathfrak{A}}^{(d+1)}$, and thus is the range of a projection $Q$ in its commutant.

Now $P^{(d+1)}$ lies in the centre of $\widetilde{\mathfrak{A}}^{(d+1)}$, and thus commutes with $Q$ as well. Therefore $\widetilde{\mathcal{V}}^{(d+1)}$ decomposes as

$$
\begin{gathered}
P^{(d+1)} Q \widetilde{\mathcal{V}}^{(d+1)} \oplus P^{\perp(d+1)} Q \widetilde{\mathcal{V}}^{(d+1)} \oplus P^{(d+1)} Q^{\perp} \widetilde{\mathcal{V}}^{(d+1)} \oplus P^{\perp(d+1)} Q^{\perp} \widetilde{\mathcal{V}}^{(d+1)} \\
=: \mathcal{M}_{p q} \oplus \mathcal{M}_{p^{\perp} q} \oplus \mathcal{M}_{p q^{\perp}} \oplus \mathcal{M}_{p^{\perp} q^{\perp}}
\end{gathered}
$$

This determines an orthogonal decomposition of $\widetilde{\mathcal{V}}^{(d+1)}$ into four reducing subspaces for $\widetilde{\mathfrak{A}}^{(d+1)}$. Note that $\mathcal{M}_{0}$ is the sum of the first two. Recall the remarks following Lemma 5.9 that $S$ is the minimal isometric dilation of $\widetilde{A}$. So by Lemma $3.4, \mathcal{H}^{(d+1)}$ has an orthogonal decomposition into the four reducing subspaces for $\mathfrak{S}^{(d+1)}$ generated by these subspaces of $\widetilde{\mathcal{V}}^{(d+1)}$, say

$$
\mathcal{H}^{(d+1)}=\mathcal{H}_{p q} \oplus \mathcal{H}_{p^{\perp} q} \oplus \mathcal{H}_{p q^{\perp}} \oplus \mathcal{H}_{p^{\perp} q^{\perp}}
$$

Moreover,the Key Lemma 4.1 shows as in the proof of Theorem 5.2 that $y$ belongs to $\mathcal{H}_{p q} \oplus \mathcal{H}_{p^{\perp} q}=\mathfrak{S}^{(d+1)}\left[\mathcal{M}_{0}\right]$.

It is evident from this construction that each of these four subspaces $\mathcal{H}_{i j}$ is mapped onto the corresponding $\mathcal{M}_{i j}$ by the orthogonal projection $P_{\widetilde{\mathcal{V}}}^{(d+1)}$ onto $\widetilde{\mathcal{V}}^{(d+1)}$. Therefore, since $P^{(d+1)}$ is dominated by this projection, it is clear that it maps $y$ into $\mathcal{M}_{p q}$, which is contained in $\mathcal{M}_{0}$. As before, we obtain that $x$ is orthogonal to $\mathcal{M}_{0}$, and therefore $\varphi(P)=0$. Hence we conclude that $P$ belongs to $\mathfrak{S}$. 
Theorem 5.15. Let $A_{1}, \ldots, A_{n}$ be operators on a finite dimensional space $\mathcal{V}$ such that $\sum_{i=1}^{n} A_{i} A_{i}^{*}=I$, and let $S_{1}, \ldots, S_{n}$ be their joint isometric dilation. Let $\widetilde{\mathcal{V}}$ be the subspace of $\mathcal{V}$ spanned by all minimal $\mathfrak{A}^{*}$-invariant subspaces. Then the compression $\widetilde{\mathfrak{A}}$ of $\mathfrak{A}$ to $\widetilde{\mathcal{V}}$ is a $C^{*}$ algebra. Let $\widetilde{\mathfrak{A}}$ be decomposed as $\sum_{g \in G}^{\oplus} \mathfrak{M}_{d_{g}} \otimes \mathbb{C}^{m_{g}}$ with respect to a decomposition $\widetilde{\mathcal{V}}=\sum_{g \in G}^{\oplus} \mathcal{V}_{g}^{\left(m_{g}\right)}$, where $\mathcal{V}_{g}$ has dimension $d_{g}$ and multiplicity $m_{g}$. Let $P_{g}$ denote the projection onto $\mathcal{V}_{g}$. Then the dilation acts on the space

$$
\mathcal{H}=\sum_{g \in G}^{\oplus} \mathcal{H}_{g}^{\left(m_{g}\right)}=\tilde{\mathcal{V}} \oplus \mathcal{K}_{n}^{(\alpha)}
$$

where $\mathcal{H}_{g}=\mathcal{V}_{g} \oplus \mathcal{K}_{n}^{\left(\alpha_{g}\right)}$ and $\alpha_{g}=d_{g}(n-1)$ and

$$
\alpha=\sum_{g \in G} \alpha_{g} m_{g}=(n-1) \sum_{g \in G} d_{g} m_{g} .
$$

The algebra $\mathfrak{S}$ decomposes as

$$
\mathfrak{S} \simeq \sum_{g \in G}^{\oplus}\left(\mathcal{B}\left(\mathcal{H}_{g}\right) P_{g}\right)^{\left(m_{g}\right)}+\left(0_{\tilde{\mathcal{V}}} \oplus \mathfrak{L}_{n}^{(\alpha)}\right)
$$

Proof. This is now just a matter of putting the pieces together and clearing up some final details. Let $\mathcal{V}_{g}, 1 \leq g \leq s$, be any maximal family of pairwise orthogonal minimal $\mathfrak{A}^{*}$-invariant subspaces. Let $\widetilde{\mathcal{V}}=$ $\sum_{1 \leq g \leq s}^{\oplus} \mathcal{V}_{g}$. (Do not worry at this stage about the uniqueness of the definition of $\widetilde{\mathcal{V}}$.) By Lemma 3.4, $\mathcal{H}_{g}=\mathfrak{S}\left[\mathcal{V}_{g}\right]$ are pairwise orthogonal reducing subspaces of $\mathfrak{S}$. Let $\mathcal{M}=\sum_{1 \leq g \leq s}^{\oplus} \mathcal{H}_{g}$. We claim that $\mathcal{M}=\mathcal{H}$. Indeed, were there a non-zero vector in $\mathcal{M}^{\perp}$, then by Corollary 4.2 of the Key Technical Lemma, $\mathcal{M}^{\perp} \cap \mathcal{V}$ would be an non-zero $\mathfrak{A}^{*}$-invariant subspace orthogonal to $\widetilde{\mathcal{V}}$, contrary to fact.

It now follows as above that if we compress each $A_{i}$ to $\widetilde{A}_{i}$ on $\widetilde{\mathcal{V}}$, then this new $n$-tuple has the identical joint isometric dilation $S_{i}$, and it is the minimal dilation by the previous paragraph. By Theorem 5.13, the algebra $\widetilde{\mathfrak{A}}$ that they generate is self-adjoint. Then applying Lemma 5.14 , we deduce that the projection onto $\widetilde{\mathcal{V}}$ belongs to $\mathfrak{S}$, and that $P_{\widetilde{\mathcal{V}}} \mathfrak{S}=\widetilde{\mathfrak{A}}$.

The restriction of $\mathfrak{S}$ to each reducing subspace $\mathcal{H}_{g}$ is isomorphic to $\mathfrak{B}_{n, d_{g}}$. Moreover the restriction of $\mathfrak{S}$ to $\widetilde{\mathcal{V}}^{\perp}$ is canonically isomorphic to $\mathfrak{L}_{n}^{(\alpha)}$, where by canonical we mean that $\left.u(S)\right|_{\tilde{\mathcal{V}}^{\perp}} \simeq L_{u}^{(\alpha)}$ when we make the natural identification of $\widetilde{\mathcal{V}}^{\perp}$ with $\mathcal{K}_{n}^{(\alpha)}$ as in Lemma 3.1. 
Now the finite dimensional $\mathrm{C}^{*}$-algebra $\widetilde{\mathfrak{A}}$ may be decomposed as $\sum_{g \in G}^{\oplus} \mathfrak{M}_{d_{g}} \otimes \mathbb{C}^{m_{g}}$. The multiplicities reflect the fact that the restrictions of $A_{i}^{*}$ to different $\mathcal{V}_{g}$ 's may be unitarily equivalent. As before, choose a maximal subset $G$ of pairwise inequivalent subspaces $\mathcal{V}_{g}$, and let $\mathcal{W}_{g}=\sum_{j \in G_{g}}^{\oplus} \mathcal{V}_{j}$ be the sum of all subspaces equivalent to $\mathcal{V}_{g}$. Then $\mathcal{W}_{g}$ may be naturally identified with $\mathcal{V}_{g} \otimes \mathbb{C}^{m_{g}}$ so that $\left.A_{i}^{*}\right|_{\mathcal{W}_{g}} \simeq\left(\left.A_{i}^{*}\right|_{\mathcal{V}_{g}}\right)^{\left(m_{g}\right)}$. This identifies $\widetilde{\mathcal{V}}$ with $\sum_{g \in G}^{\oplus} \mathcal{V}_{g}^{\left(m_{g}\right)}$.

By the uniqueness of the minimal isometric dilation, it also follows that there is a corresponding unitary equivalence between $\sum_{j \in G_{g}}^{\oplus} \mathcal{H}_{j}$ and $\mathcal{H}_{g} \otimes \mathbb{C}^{\left(m_{g}\right)}$ so that the restriction of $S_{i}$ is identified with $\left(\left.S_{i}\right|_{\mathcal{H}_{g}}\right)^{\left(m_{g}\right)}$. By Lemma 5.14, the projection $P_{\mathcal{W}_{g}} \simeq P_{g}^{\left(m_{g}\right)}$ belongs to $\mathfrak{S}$. Thus we now see that $\mathfrak{S} P_{\widetilde{\mathcal{V}}}$ decomposes as $\sum_{g \in G}^{\oplus}\left(\mathcal{B}\left(\mathcal{H}_{g}\right) P_{g}\right)^{\left(m_{g}\right)}$. Combining all of the pieces, we obtain the desired structure theory for $\mathfrak{S}$.

It remains to establish the uniqueness of $\widetilde{\mathcal{V}}$. We can now see that $P_{\widetilde{\mathcal{V}}}$ is the unique maximal finite rank projection in $\mathfrak{S}$. Indeed, every operator in $\mathfrak{S}$ has a lower triangular form with respect to the decomposition $\mathcal{H}=\widetilde{\mathcal{V}} \oplus \mathcal{K}_{n}^{(\alpha)}$. By [15, Corollary 1.8], $\mathfrak{L}_{n}$ contains no proper projections. Therefore all finite rank projections are supported by $\widetilde{\mathcal{V}}$. Now suppose that $\mathcal{V}_{0}$ is any minimal $\mathfrak{A}^{*}$-invariant subspace. It may be extended to a maximal family of pairwise orthogonal minimal $\mathfrak{A}^{*}$-invariant subspaces, and the construction may proceed as above. The same subspace $\widetilde{\mathcal{V}}$ necessarily is obtained by the uniqueness of this maximal projection. In particular, $\widetilde{\mathcal{V}}$ must contain every minimal $\mathfrak{A}^{*}$-invariant subspace. Thus it is the span of all such subspaces.

\section{The General Finite Dimensional Case}

We now return to the problem posed in Section 4. Starting with a contractive $n$-tuple $A_{1}, \ldots, A_{n}$ with minimal joint isometric dilation $S_{1}, \ldots, S_{n}$, we wish to understand the structure of $\mathfrak{S}=\operatorname{Alg}\left\{S_{1}, \ldots, S_{n}\right\}$ in terms of the structure of the $n$-tuple $A$ and the algebra $\mathfrak{A}$ that it generates.

Recall from the discussion in Section 4 that $\mathcal{H}=\mathcal{H}_{p} \oplus \mathcal{H}_{c}$, where $\mathcal{H}_{p}$ is the pure part determined by the wandering subspace of $S$, and $\mathcal{H}_{c}$ is the Cuntz part; and that $P_{p}$ and $P_{c}$ denote the orthogonal projections onto these subspaces. We need a method of getting information about this decomposition from $A$. Corollary 4.2 of the Key Technical Lemma shows that $\mathcal{H}_{c}=\mathfrak{S}\left[\mathcal{V}_{c}\right]$, so $\mathcal{H}_{c}$ is recovered if we can compute $\mathcal{V}_{c}$.

Again we consider the completely positive map $\Phi(X)=\sum_{i=1}^{n} A_{i} X A_{i}^{*}$. This is no longer unital, since $\Phi(I)=A A^{*}=\sum_{i=1}^{n} A_{i} A_{i}^{*} \leq I$. But it 
is completely contractive. Thus the sequence $\Phi^{k}(I)$ is a decreasing sequence of positive operators, and therefore converges to a limit which we denote as $\Phi^{\infty}(I)$.

Lemma 6.1. $\Phi^{\infty}(I)=P_{\mathcal{V}} P_{c} P_{\mathcal{V}}$. Hence $\mathcal{V}_{c}=\operatorname{ker}\left(I-\Phi^{\infty}(I)\right)$.

Proof. If $x \in \mathcal{H}_{c}$,

$$
\sum_{|w|=k}\left\|S_{w}^{*} x\right\|^{2}=\|x\|^{2} .
$$

On the other hand, any vector $x$ in $\mathcal{H}_{p}$ satisfies

$$
\lim _{k \rightarrow \infty} \sum_{|w|=k}\left\|S_{w}^{*} x\right\|^{2}=0 .
$$

Thus if $x$ is any vector in $\mathcal{H}=\mathcal{H}_{c} \oplus \mathcal{H}_{p}$,

$$
\lim _{k \rightarrow \infty} \sum_{|w|=k}\left\|S_{w}^{*} x\right\|^{2}=\left\|P_{c} x\right\|^{2} .
$$

We write $A_{w}^{*}:=w(A)^{*}=S_{w}^{*} \mid \mathcal{V}$. Now if $v \in \mathcal{V}$,

$$
\lim _{k \rightarrow \infty} \sum_{|w|=k}\left\|A_{w}^{*} v\right\|^{2}=\lim _{k \rightarrow \infty} \sum_{|w|=k}\left\|S_{w}^{*} v\right\|^{2}=\left\|P_{c} v\right\|^{2} .
$$

It is evident that $\Phi^{k}(I)=\sum_{|w|=k} A_{w} A_{w}^{*}$ and thus

$$
\left(\Phi^{k}(I) v, v\right)=\sum_{|w|=k}\left\|A_{w}^{*} v\right\|^{2} .
$$

Therefore

$$
\left(\Phi^{\infty}(I) v, v\right)=\left\|P_{c} v\right\|^{2}=\left(P_{\mathcal{V}} P_{c} P_{\mathcal{V}} v, v\right)
$$

Since a sesquilinear form can be recovered from its quadratic form by the polarization identity, it follows that $\Phi^{\infty}(I)=P_{\mathcal{V}} P_{c} P_{\mathcal{V}}$.

In particular, $\operatorname{ker}\left(I-\Phi^{\infty}(I)\right)=\mathcal{V} \cap \mathcal{H}_{c}=\mathcal{V}_{c}$.

We have $\mathcal{H}_{c}=\mathfrak{S}\left[\mathcal{V}_{c}\right]$, and thus the restriction of the $S_{i}$ 's to $\mathcal{H}_{c}$ are the minimal joint isometric dilations of the compressions of the $A_{i}$ 's to $\mathcal{V}_{c}$. By the previous section, we know that $\left.\mathfrak{S}\right|_{\mathcal{H}_{c}}$ is determined by the restriction of $\mathfrak{A}$ to the span $\widetilde{\mathcal{V}}$ of all $\mathfrak{A}^{*}$-invariant subspaces contained in $\mathcal{V}_{c}$. It is desirable to give a definition that is somewhat independent of the definition of $\mathcal{V}_{c}$. the space $\widetilde{\mathcal{V}}$ is the span of all $\mathfrak{A}^{*}$-invariant subspaces $\mathcal{W}$ on which $\left.\sum_{i=1}^{n} A_{i} A_{i}^{*}\right|_{\mathcal{W}}=I_{\mathcal{W}}$. Indeed, the condition

$$
I_{\mathcal{W}}=\left.\sum_{i=1}^{n} A_{i} A_{i}^{*}\right|_{\mathcal{W}}=\left.\sum_{i=1}^{n} S_{i} S_{i}^{*}\right|_{\mathcal{W}}
$$


implies that $\mathcal{W}$ is contained in $\mathcal{H}_{c}$, whence in $\mathcal{H}_{c} \cap \mathcal{V}=\mathcal{V}_{c}$. Thus $\mathcal{W}$ is contained in $\widetilde{\mathcal{V}}$ by Theorem 5.15. The converse follows from the description there of $\widetilde{\mathcal{V}}$.

Lemma 6.2. The projection $P_{\widetilde{\mathcal{V}}}$ belongs to $\mathfrak{S}$.

Proof. We may assume that $P_{\widetilde{\mathcal{V}}} \neq 0$. Decompose $\mathcal{H}$ as $\mathcal{H}_{c} \oplus \mathcal{H}_{p}$. Let $\mathfrak{S}_{c}$ denote the restriction of $\mathfrak{S}$ to $\mathcal{H}_{c}$. By the Cuntz case, Theorem 5.14, the projection $P_{\widetilde{\mathcal{V}}}$ belongs to the WOT-closure of $\mathfrak{S}_{c}$. In other words there is a net $A_{\alpha}$ of polynomials in $S$ such that $\left.A_{\alpha}\right|_{\mathcal{H}_{c}}$ converges in the WOT topology to $P_{\widetilde{\mathcal{V}}}$. Since $\mathcal{H}_{c}$ contains a wandering vector $\xi$, the subspace $\mathfrak{S}[\xi]$ is unitarily equivalent to $\mathfrak{L}_{n}$. Moreover the restriction of $A_{\alpha}$ to $\mathfrak{S}[\xi]$ converges weakly to 0 . Now the restriction of $A_{\alpha}$ to $\mathcal{H}_{p}$ is unitarily equivalent to a multiple of $\left.A_{\alpha}\right|_{\mathfrak{S}[\xi]}$, and thus it also converges weakly to 0 . Combining the two parts, we see that $A_{\alpha}$ converges to $P_{\widetilde{\mathcal{V}}}$ in $\mathcal{B}(\mathcal{H})$.

Next we wish to compute the pure rank of the dilation. Notice that the proof which follows does not require that the $n$-tuple act on a finite dimensional space. This fact is used in the development of the non-commutative curvature invariant and Euler characteristic in [24].

Lemma 6.3. The pure rank of $\mathfrak{S}$ is computed as

$$
\operatorname{pure} \operatorname{rank}(\mathfrak{S})=\operatorname{rank}(I-\Phi(I))=\operatorname{rank}\left(I-\sum_{i=1}^{n} A_{i} A_{i}^{*}\right) .
$$

Proof. The wandering space is $\mathcal{X}=\operatorname{Ran}\left(I-\sum_{i=1}^{n} S_{i} S_{i}^{*}\right)$ and the pure rank of $\mathfrak{S}$ equals $\operatorname{dim} \mathcal{X}$. The minimality of the dilation means that $\mathcal{X}$ does not intersect $\mathcal{V}^{\perp}$. Therefore $P_{\mathcal{V}} P_{\mathcal{X}} P_{\mathcal{V}}$ has the same rank as $P_{\mathcal{X}}$. However it is easy to see that

$$
\begin{aligned}
P_{\mathcal{V}} P_{\mathcal{X}} P_{\mathcal{V}} \mid \mathcal{V} & =\left.P_{\mathcal{V}}\left(I_{\mathcal{H}}-\sum_{i=1}^{n} S_{i} S_{i}^{*}\right) P_{\mathcal{V}}\right|_{\mathcal{V}} \\
& =I_{\mathcal{V}}-\sum_{i=1}^{n} A_{i} A_{i}^{*}=I_{\mathcal{V}}-\Phi\left(I_{\mathcal{V}}\right)
\end{aligned}
$$

Thus pure $\operatorname{rank}(\mathfrak{S})=\operatorname{rank}(I-\Phi(I))$.

Example 6.4. Any subtlety of the preceding lemma is due to fact that $\mathcal{X}$ is not, in general, contained in $\mathcal{V}$. To illustrate this, consider the following example. Let

$$
A_{1}=\left[\begin{array}{lll}
1 & 0 & 0 \\
0 & 0 & 0 \\
0 & 0 & 0
\end{array}\right] \quad \text { and } \quad A_{2}=\left[\begin{array}{ccc}
0 & 0 & 0 \\
1 / 2 & 0 & 1 / 2 \\
0 & 0 & 0
\end{array}\right]
$$


Then

$$
A_{1} A_{1}^{*}+A_{2} A_{2}^{*}=\left[\begin{array}{ccc}
1 & 0 & 0 \\
0 & 1 / 2 & 0 \\
0 & 0 & 0
\end{array}\right] .
$$

It is clear that $\mathbb{C} e_{1}$ and $\mathbb{C} e_{3}$ are pairwise orthogonal minimal $\mathfrak{A}^{*}$-invariant subspaces. The vector $e_{1}$ generates the subspace $\mathcal{H}_{1}=\overline{\mathfrak{S} e_{1}}$ on which the representation is equivalent to the atomic representation $\sigma_{1,1}$. Furthermore, $e_{3}$ is a wandering vector generating a copy of the left regular representation on $\mathcal{H}_{3}=\overline{\mathfrak{S} e_{3}}$. However $e_{2}$ is not orthogonal to $\mathcal{H}_{1} \oplus \mathcal{H}_{3}$. One can show that there is a second wandering vector $\zeta:=$ $e_{2}-P_{\mathcal{V}}^{\perp} S_{2}\left(e_{1}+e_{3}\right)$. The subspace $\mathcal{H}_{2}=\overline{\mathfrak{S} \zeta}$ yields the decomposition $\mathcal{H}=\mathcal{H}_{1} \oplus \mathcal{H}_{2} \oplus \mathcal{H}_{3}$.

The point here is that this decomposition does not decompose $\mathcal{V}$ into orthogonal pieces. In fact, $\mathcal{H}_{2}$ has trivial intersection with $\mathcal{V}$; and the vector $e_{2}$ has components in all three pieces.

We can now completely describe the algebra $\mathfrak{S}$ determined by the joint isometric dilation of a contractive $n$-tuple. There is nothing to do except combine the information in Theorem 5.15 with the preceding two lemmas.

Theorem 6.5. Let $A_{1}, \ldots, A_{n}$ be a contractive $n$-tuple on a finite dimensional space $\mathcal{V}$ with joint minimal isometric dilation $S_{1}, \ldots, S_{n}$ on $\mathcal{H}$. The space $\mathcal{H}$ decomposes as $\mathcal{H}_{p} \oplus \mathcal{H}_{c}$ into its pure and Cuntz parts. The multiplicity of $\mathcal{H}_{p}$ is pure $\operatorname{rank}(\mathfrak{S})=\operatorname{rank}\left(I-\sum_{i=1}^{n} A_{i} A_{i}^{*}\right)$. The subspace $\widetilde{\mathcal{V}}$ spanned by all minimal $\mathfrak{A}^{*}$-invariant subspaces $\mathcal{W}$ on which $\left.\sum_{i=1}^{n} A_{i} A_{i}^{*}\right|_{\mathcal{W}}=I_{\mathcal{W}}$ determines $\mathcal{H}_{c}=\mathfrak{S}[\widetilde{\mathcal{V}}]$

The compression $\widetilde{\mathfrak{A}}$ of $\mathfrak{A}$ to $\widetilde{\mathcal{V}}$ is a $C^{*}$-algebra. Let $\widetilde{\mathfrak{A}}$ be decomposed as $\sum_{g \in G}^{\oplus} \mathfrak{M}_{d_{g}} \otimes \mathbb{C}^{m_{g}}$ with respect to a decomposition $\widetilde{\mathcal{V}}=\sum_{g \in G}^{\oplus} \mathcal{V}_{g}^{\left(m_{g}\right)}$, where $\mathcal{V}_{g}$ has dimension $d_{g}$ and multiplicity $m_{g}$; and let $P_{g}$ denote the projection onto $\mathcal{V}_{g}$. Then the dilation acts on the space

$$
\mathcal{H}=\sum_{g \in G}^{\oplus} \mathcal{H}_{g}^{\left(m_{g}\right)} \oplus \mathcal{H}_{p}=\widetilde{\mathcal{V}} \oplus \mathcal{K}_{n}^{(\alpha)}
$$

where $\mathcal{H}_{g}=\mathcal{V}_{g} \oplus \mathcal{K}_{n}^{\left(\alpha_{g}\right)}, \alpha_{g}=d_{g}(n-1)$ and

$$
\begin{aligned}
\alpha & =\sum_{g \in G} \alpha_{g} m_{g}+\operatorname{pure} \operatorname{rank}(\mathfrak{S}) \\
& =(n-1) \sum_{g \in G} d_{g} m_{g}+\operatorname{rank}\left(I-\sum_{i=1}^{n} A_{i} A_{i}^{*}\right) .
\end{aligned}
$$


The algebra $\mathfrak{S}$ decomposes as

$$
\mathfrak{S} \simeq \sum_{g \in G}^{\oplus}\left(\mathcal{B}\left(\mathcal{H}_{g}\right) P_{g}\right)^{\left(m_{g}\right)}+\left(0_{\tilde{\mathcal{V}}} \oplus \mathfrak{L}_{n}^{(\alpha)}\right) .
$$

We now collect some of the consequences of this theorem. First we obtain simple conditions to determine when the dilation of $A$ is irreducible.

Corollary 6.6. The algebra $\mathfrak{S}$ determined by the joint isometric dilation of a contractive $n$-tuple $A$ on a finite dimensional space $\mathcal{V}$ is irreducible if and only if either

(1) $\operatorname{Ran}\left(I-\sum_{i=1}^{n} A_{i} A_{i}^{*}\right)=\mathbb{C} v \neq 0$ and $v$ is cyclic for $\mathfrak{A}$. In this case, $\mathfrak{S}$ is unitarily equivalent to $\mathfrak{L}_{n}$.

or

(2) $\sum_{i=1}^{n} A_{i} A_{i}^{*}=I$ and $\mathfrak{A}^{*}$ has a minimal invariant subspace $\mathcal{V}_{0}$ which is cyclic for $\mathfrak{A}$. In this case, $\mathfrak{S}$ is unitarily equivalent to $\mathfrak{B}_{n, d_{0}}$ where $d_{0}=\operatorname{dim} \mathcal{V}_{0}$.

which are respectively equivalent to

$\left(1^{\prime}\right) \operatorname{rank}(I-\Phi(I))=1$ and $\Phi^{\infty}(I)=0$.

or

$\left(2^{\prime}\right)\{X: \Phi(X)=X\}=\mathbb{C} I$.

Proof. $\mathfrak{S}$ is irreducible if and only if either it is pure with pure rank 1 , or it has pure rank 0 and, by Lemma 5.8, has a unique minimal $\mathfrak{A}^{*}$-invariant subspace.

By Lemma 6.3, the pure rank is 1 precisely when $\operatorname{rank}(I-\Phi(I))=1$, or equivalently that $\operatorname{Ran}\left(I-\sum_{i=1}^{n} A_{i} A_{i}^{*}\right)$ is a one-dimensional subspace $\mathbb{C} v$. Now $\mathfrak{S}$ is pure precisely when $\mathcal{H}_{c}=\{0\}$, which by Corollary 4.2 is equivalent to $\mathcal{V}_{c}=\{0\}$. By Lemma 6.1, this is equivalent to $\Phi^{\infty}(I)=0$, which establishes the equivalence with $\left(1^{\prime}\right)$. Now $\mathcal{V}_{c}$ is $\mathfrak{A}^{*}$-invariant and orthogonal to $v$, and therefore orthogonal to $\mathfrak{A} v$. So if $v$ is $\mathfrak{A}-$ cyclic, then $\mathfrak{A}[v]=\mathcal{V}$ and $\mathcal{V}_{c}=\{0\}$. Conversely, if $\mathfrak{A}[v]$ is proper, then $\mathcal{M}=\mathfrak{A}[v]^{\perp}$ is $\mathfrak{A}^{*}$-invariant. But $\left.\sum_{i=1}^{n} A_{i} A_{i}^{*}\right|_{\mathcal{M}}=I_{\mathcal{M}}$ because of the condition on $\Phi(I)$. So $\mathcal{M}$ is contained in $\mathcal{H}_{c}$. This verifies the equivalence with (1).

The Cuntz case is synonymous with the condition $\sum_{i=1}^{n} A_{i} A_{i}^{*}=I$. If $\mathcal{M}$ is a minimal $\mathfrak{A}^{*}$-invariant subspace, then $\mathfrak{A}[\mathcal{M}]^{\perp}$ contains another. So if $\mathcal{M}$ is unique, it must be cyclic. Conversely, if it is not unique, then by Theorem $5.15, \widetilde{\mathcal{V}}$ contains at least two pairwise orthogonal minimal $\mathfrak{A}^{*}$-invariant subspaces, one of which may be taken to be $\mathcal{M}$; call the other $\mathcal{M}^{\prime}$. Then $\mathfrak{A}[\mathcal{M}]$ is orthogonal to $\mathcal{M}^{\prime}$ and thus it is not cyclic for $\mathfrak{A}$. This establishes the equivalence with (2). 
Condition $\left(2^{\prime}\right)$ contains the fact that $\Phi(I)=I$, so this is the Cuntz case. If there were more than one minimal $\mathfrak{A}^{*}$-invariant subspace, then by Theorem 5.13 the fixed point algebra contains non-scalar operators. Conversely, if $\Phi$ has non-scalar fixed points, then Lemma 5.10 shows that there are two orthogonal $\mathfrak{A}^{*}$-invariant subspaces. So $\left(2^{\prime}\right)$ is equivalent to irreducibility.

Corollary 6.7. The minimal isometric dilation of a finite dimensional $n$-tuple $A=\left(A_{1}, \ldots, A_{n}\right)$ is pure if and only if $\mathfrak{A}\left(I-\sum_{i=1}^{n} A_{i} A_{i}^{*}\right) \mathcal{V}=\mathcal{V}$ or equivalently that $\Phi^{\infty}(I)=0$.

Proof. The dilation has a Cuntz part if and only if there is a $\mathfrak{A}^{*}$ invariant subspace $\mathcal{M}$ contained in $\operatorname{ker}\left(I-\sum_{i=1}^{n} A_{i} A_{i}^{*}\right)$. This is equivalent to having the proper $\mathfrak{A}$-invariant subspace $\mathcal{M}^{\perp}$ containing

$$
\left(\operatorname{ker}\left(I-\sum_{i=1}^{n} A_{i} A_{i}^{*}\right)\right)^{\perp}=\operatorname{Ran}\left(I-\sum_{i=1}^{n} A_{i} A_{i}^{*}\right) .
$$

The minimal such subspace is clearly $\mathfrak{A}\left(I-\sum_{i=1}^{n} A_{i} A_{i}^{*}\right) \mathcal{V}$. Thus the dilation is pure precisely when $\mathfrak{A}\left(I-\sum_{i=1}^{n} A_{i} A_{i}^{*}\right) \mathcal{V}=\mathcal{V}$.

Evidently, if there is a Cuntz part, then

$$
\Phi^{\infty}(I) \geq \Phi^{\infty}\left(P_{\widetilde{\mathcal{V}}}\right)=P_{\widetilde{\mathcal{V}}}
$$

Conversely, if $A$ is pure, then $\mathrm{SOT}-\lim _{k \rightarrow \infty} \sum_{|w|=k} S_{w} S_{w}^{*}=0$. The compression of $S_{w} S_{w}^{*}$ to $\mathcal{V}$ is $A_{w} A_{w}^{*}$, and thus

$$
\sum_{|w|=k} P_{\mathcal{V}} S_{w} S_{w}^{*} \mid \mathcal{V}=\sum_{|w|=k} A_{w} A_{w}^{*}=\Phi^{k}(I) .
$$

Since $\mathcal{V}$ is finite dimensional, this converges to 0 in norm.

Our theorem also provides simple complete unitary invariants for the associated finitely correlated representations of $\mathcal{E}_{n}$ (or of $\mathcal{O}_{n}$ in the Cuntz case).

Theorem 6.8. Let $A=\left(A_{1}, \ldots, A_{n}\right)$ and $B=\left(B_{1}, \ldots, B_{n}\right)$ be contractive $n$-tuples on finite dimensional spaces $\mathcal{V}_{A}$ and $\mathcal{V}_{B}$ respectively. Let $S=\left(S_{1}, \ldots, S_{n}\right)$ and $T=\left(T_{1}, \ldots, T_{n}\right)$ be their joint minimal isometric dilations on Hilbert spaces $\mathcal{H}_{A}$ and $\mathcal{H}_{B}$; and let $\sigma_{A}$ and $\sigma_{B}$ be the induced representations of $\mathcal{E}_{n}$. Let $\widetilde{\mathcal{V}}_{A}$ be the subspace spanned by all minimal $\mathfrak{A}^{*}$-invariant subspaces $\mathcal{W}$ on which $\left.\sum_{i=1}^{n} A_{i} A_{i}^{*}\right|_{\mathcal{W}}=I_{\mathcal{W}}$; and similarly define $\widetilde{\mathcal{V}}_{B}$. Then $\sigma_{A}$ and $\sigma_{B}$ are unitarily equivalent if and only if

(1) $\operatorname{rank}\left(I_{\mathcal{V}_{A}}-\sum_{i=1}^{n} A_{i} A_{i}^{*}\right)=\operatorname{rank}\left(I_{\mathcal{V}_{B}}-\sum_{i=1}^{n} B_{i} B_{i}^{*}\right)$; and

(2) $\left.A^{*}\right|_{\widetilde{\mathcal{V}}_{A}}$ is unitarily equivalent to $\left.B^{*}\right|_{\widetilde{\mathcal{V}}_{B}}$. 
Proof. The two representations are equivalent if and only if they have the same pure rank and the Cuntz parts are unitarily equivalent. By Theorem 6.5 , the algebra $\mathfrak{S}$ contains the projection onto $\widetilde{\mathcal{V}}_{A}$. It is the unique maximal finite rank projection in $\mathfrak{S}$. Therefore the restriction $\left.A^{*}\right|_{\widetilde{\mathcal{V}}_{A}}$ is a unitary invariant. Conversely, if these two conditions hold, then the unitary identifying $\left.A^{*}\right|_{\widetilde{\mathcal{V}}_{A}}$ and $\left.B^{*}\right|_{\widetilde{\mathcal{V}}_{B}}$ extends to a unitary equivalence between the dilations $S_{A}$ of $\widetilde{A}:=\left.P_{\widetilde{\mathcal{V}}_{A}} A\right|_{\widetilde{\mathcal{V}}_{A}}$ and $S_{B}$ of $\widetilde{B}:=$ $\left.P_{\widetilde{\mathcal{V}}_{B}} B\right|_{\widetilde{\mathcal{V}}_{B}}$ because of the uniqueness of the minimal isometric dilation. This identifies the restriction of $S_{A}$ to $\mathfrak{S}\left[\widetilde{\mathcal{V}}_{A}\right]=\mathcal{H}_{A c}$, namely the Cuntz part of $S_{A}$, with the corresponding Cuntz part of $S_{B}$. The pure rank condition allows a unitary equivalence between the two pure parts.

Bratteli and Jorgensen [9] give a detailed analysis of representations of the Cuntz algebra which has a lot in common with our results. They look somewhat different since they concentrate on the state and not on the restriction to the subspace $\mathcal{V}$. In particular, their contractions are not the same as ours. They point out the relationship in the discussion preceding their Theorem 5.3. They obtain our Corollary 6.6 in the Cuntz case, and in particular recognize the role of the completely positive map $\Phi$. Again however, their different normalization results in a different map. But they do not appear to classify these representations up to unitary equivalence. The reason they do not succeed is that they did not identify the subspace which we call $\widetilde{\mathcal{V}}$, and instead work with a subspace they call $\mathcal{V}_{k}$ which is often strictly larger. The space $\widetilde{\mathcal{V}}$ does not occur in their hierarchy of invariant subspaces. Instead, they specialize in section 7 to a smaller class which they call diagonalizable shifts. These they do completely classify up to unitary equivalence. We have not determined in this case how their special invariants relate to ours.

Corollary 6.9. The algebra $\mathfrak{S}$ determined by the joint isometric dilation of a contractive $n$-tuple on a finite dimensional space is hyperreflexive with distance constant at most 5.

Proof. This follows immediately from [15, Theorem 3.14] since the algebra $\mathfrak{S}$ is unitarily equivalent to the algebra of certain atomic representations. Indeed, the projection $P=P_{\widetilde{\mathcal{V}}}$ belongs to $\mathfrak{S}$ and $\mathfrak{S} P=\mathfrak{W} P$ where $\mathfrak{W}$ is a type I von Neumann algebra containing the projection $P$. Thus by Christensen's result [12] which shows that type I von Neumann algebras have distance constant at most 4 , we obtain the same for our slice. The upper bound for the distance constant of $\mathfrak{L}_{n}$ was improved by Bercovici [6] to 3 from the original 51. Arguing as in [15], we obtain a distance constant no larger than $\left(3^{2}+4^{2}\right)^{1 / 2}=5$. 


\section{Similarity}

Now consider the question of when two contractive $n$-tuples are similar, and the effect on their dilations. The first step is to show that the Cuntz parts must be unitarily equivalent. Thus the question of similarity reduces to the pure parts. First we need a variant of Lemma 5.10.

Lemma 7.1. Suppose that an $n$-tuple $\left(A_{1}, \ldots, A_{n}\right)$ acts on a finite dimensional space $\mathcal{V}$, and generates $\mathcal{B}(\mathcal{V})$ as an algebra. Moreover suppose that $\Phi(X)=\sum_{i=1}^{n} A_{i} X A_{i}^{*}$ is unital. Then the only self-adjoint operators $X$ satisfying $\Phi(X) \leq X$ are scalar, and in particular are fixed points.

Proof. Since $\Phi(I)=I$, we may translate $X$ so that $X \geq 0$ and 0 belongs to its spectrum. Let $\mathcal{M}=\operatorname{ker} X$. This is a non-zero subspace. Let $x \in \mathcal{M}$. Then

$$
\begin{aligned}
0 & =(\Phi(0) x, x) \leq(\Phi(X) x, x) \\
& =\sum_{i=1}^{n}\left(A_{i} X A_{i}^{*} x, x\right)=\sum_{i=1}^{n}\left\|X A_{i}^{*} x\right\|^{2} \leq(X x, x)=0 .
\end{aligned}
$$

It follows that $\mathcal{M}$ is invariant for each $A_{i}^{*}$. But by hypothesis, the $A_{i}^{*}$ 's generate the full matrix algebra, and thus have no proper invariant subspaces. So $\mathcal{M}=\mathcal{V}$ and $X=0$ is scalar.

Corollary 7.2. Suppose that $A=\left(A_{1}, \ldots, A_{n}\right)$ and $B=\left(B_{1}, \ldots, B_{n}\right)$ are similar contractive $n$-tuples in the finite dimensional algebra $\mathcal{B}(\mathcal{V})$. Let $\widetilde{\mathcal{V}}_{A}$ and $\widetilde{\mathcal{V}}_{B}$ denote the subspaces spanned by the minimal $\mathfrak{A}^{*}$ and $\mathfrak{B}^{*}$-invariant subspaces $\mathcal{M}$ on which $\left.A A^{*}\right|_{\mathcal{M}}=I_{\mathcal{M}}$ and $\left.B B^{*}\right|_{\mathcal{M}}=I_{\mathcal{M}}$, respectively. Then $\left.P_{\widetilde{\mathcal{V}}_{A}} A\right|_{\widetilde{\mathcal{V}}_{A}}$ and $\left.P_{\widetilde{\mathcal{V}}_{B}} B\right|_{\widetilde{\mathcal{V}}_{B}}$ are unitarily equivalent.

Proof. Let $T$ be the similarity such that $B=T A T^{-1}$. Then $B^{*}=$ $T^{*-1} A^{*} T^{*}$. So $T^{*-1}$ carries $\mathfrak{A}^{*}$-invariant subspaces onto $\mathfrak{B}^{*}$-invariant subspaces. Also $T^{*-1}$ preserves minimality. However it is not immediately evident that it preserves the condition that $\left.A A^{*}\right|_{\tilde{\mathcal{V}}_{A}}=I_{\widetilde{\mathcal{V}}_{A}}$.

Let $\mathcal{M}$ be a minimal $\mathfrak{A}^{*}$-invariant subspace of $\widetilde{\mathcal{V}}_{A}$ on which $\left.A A^{*}\right|_{\mathcal{M}}=$ $I_{\mathcal{M}}$. Then $T^{*-1} \mathcal{M}=\mathcal{N}$ is invariant for $\mathfrak{B}^{*}$. It follows that if $\bar{A}_{i}^{*}$, and $\bar{T}^{*-1}$ are the restrictions of $A_{i}^{*}$ and $T^{*-1}$ to $\mathcal{M}$, and $\bar{B}_{i}^{*}$ is the restriction of $B_{i}^{*}$ to $\mathcal{N}$, then $\bar{B}_{i}^{*}=\bar{T}^{*-1} \bar{A}_{i}^{*} \bar{T}^{*}$. Let $\bar{\Phi}(X)=\sum_{i=1}^{n} \bar{A}_{i} X \bar{A}_{i}^{*}$ on $\mathcal{B}(\mathcal{M})$. It is easy to verify that $\bar{\Phi}$ is unital.

Now compute that

$$
I_{\mathcal{N}} \geq \sum_{i=1}^{n} \bar{B}_{i} \bar{B}_{i}^{*}=\sum_{i=1}^{n} \bar{T} \bar{A}_{i} \bar{T}^{-1} \bar{T}^{*-1} \bar{A}_{i}^{*} \bar{T}^{*}=\bar{T} \Phi\left(\bar{T}^{-1} \bar{T}^{*-1}\right) \bar{T}^{*} .
$$


Therefore $\Phi\left(\bar{T}^{-1} \bar{T}^{*-1}\right) \leq \bar{T}^{-1} \bar{T}^{*-1}$. By Lemma 7.1, it follows that $\bar{T}^{-1} \bar{T}^{*-1}$ is scalar. So up to a scaling factor, $\bar{T}$ is unitary.

This shows that the restrictions of $\mathfrak{A}^{*}$ to each minimal $\mathfrak{A}^{*}$-invariant subspace $\mathcal{M}$ of $\widetilde{\mathcal{V}}_{A}$ on which $\left.A A^{*}\right|_{\mathcal{M}}=I_{\mathcal{M}}$ is unitarily equivalent to the corresponding subspace of $\mathfrak{B}^{*}$. Since $\widetilde{\mathcal{V}}_{A}$ and $\widetilde{\mathcal{V}}_{B}$ are each the orthogonal direct sum of such subspaces, it follows that the restriction to these larger subspaces are unitarily equivalent (although $T$ itself need not be a multiple of a unitary on the whole space). Thus $\left.A^{*}\right|_{\widetilde{\mathcal{V}}_{A}}$ is unitarily equivalent to $\left.B^{*}\right|_{\widetilde{\mathcal{V}}_{B}}$. Equivalently, the compressions $\left.P_{\widetilde{\mathcal{V}}_{A}} A\right|_{\tilde{\mathcal{V}}_{A}}$ and $\left.P_{\widetilde{\mathcal{V}}_{B}} B\right|_{\widetilde{\mathcal{V}}_{B}}$ are unitarily equivalent.

Corollary 7.3. Suppose that $A=\left(A_{1}, \ldots, A_{n}\right)$ and $B=\left(B_{1}, \ldots, B_{n}\right)$ are similar contractive $n$-tuples in the finite dimensional algebra $\mathcal{B}(\mathcal{V})$. Let $S_{i}$ and $T_{i}$ be their respective minimal joint isometric dilations. Then the Cuntz parts of $S_{i}$ and $T_{i}$ are unitarily equivalent.

Proof. This is immediate from the proposition above and the fact that the Cuntz part of $S_{i}$ and $T_{i}$ are determined by the compressions of $A_{i}$ and $B_{i}$ to the subspaces $\widetilde{\mathcal{V}}_{A}$ and $\widetilde{\mathcal{V}}_{B}$ respectively by Corollary 6.8 .

Example 7.4. Now we show through a couple of examples that the pure part of the dilation is not preserved by similarity. This first example shows that one dilation can be strictly Cuntz type while a similarity can introduce a pure part. Consider

$$
A_{1}=\left[\begin{array}{ll}
1 & 0 \\
0 & 0
\end{array}\right] \quad \text { and } \quad A_{2}=\left[\begin{array}{ll}
0 & 0 \\
1 & 0
\end{array}\right] .
$$

This is of Cuntz type since $A_{1} A_{1}^{*}+A_{2} A_{2}^{*}=I$. Moreover there is a unique minimal $\mathfrak{A}^{*}$-invariant subspace, $\mathbb{C} e_{1}$. The dilation of this pair is thus irreducible by Corollary 6.6, and is determined by the 1-dimensional restrictions 1 and 0 of $A_{1}^{*}$ and $A_{2}^{*}$ to $\mathbb{C} e_{1}$. In fact, this is easily seen to be the atomic representation $\sigma_{1,1}$.

However, this pair is similar via $T=\left[\begin{array}{cc}1 & 0 \\ 0 & 1 / 2\end{array}\right]$ to

$$
B_{1}=\left[\begin{array}{ll}
1 & 0 \\
0 & 0
\end{array}\right] \quad \text { and } \quad B_{2}=\left[\begin{array}{cc}
0 & 0 \\
1 / 2 & 0
\end{array}\right] .
$$

The restrictions of $B_{i}^{*}$ to the unique minimal $\mathfrak{B}^{*}$-invariant subspace are still 1 and 0 respectively; and they determine a dilation which has the representation $\sigma_{1,1}$ as a summand. However, since

$$
\operatorname{rank}\left(I-B_{1} B_{1}^{*}-B_{2} B_{2}^{*}\right)=\operatorname{rank}\left[\begin{array}{cc}
0 & 0 \\
0 & 3 / 4
\end{array}\right]=1,
$$


the pure rank of this representation is 1 .

Example 7.5. A second easy example shows that even in the pure case, the pure rank is not a similarity invariant. Fix an orthonormal basis $e_{1}, \ldots, e_{n}$ for $\mathcal{V}$. Let $A_{1}=\frac{1}{2} e_{1} e_{1}^{*}$ and $A_{i}=e_{i} e_{1}^{*}$ for $2 \leq i \leq n$. Then $I-\sum_{i=1}^{n} A_{i} A_{i}^{*}=\frac{3}{4} e_{1} e_{1}^{*}$ is rank 1 , and its range $\mathbb{C} e_{1}$ is $\mathfrak{A}$-cyclic. So by Corollary 6.6 , this yields an irreducible pure dilation.

However, this is similar via $T=I+e_{1} e_{1}^{*}$ to $B_{1}=A_{1}$ and $B_{i}=\frac{1}{2} A_{i}$ for $2 \leq i \leq n$. This $n$-tuple satisfies $I-\sum_{i=1}^{n} B_{i} B_{i}^{*}=\frac{3}{4} I$, which has rank $n$. So this dilation has pure rank $n$.

We wish to provide more detail about the effect of similarity on pure representations. By Popescu[26], the dilation is pure if and only if

$$
\underset{k \rightarrow \infty}{\mathrm{WOT}-\lim } \sum_{|w|=k} A_{w} A_{w}^{*}=0 .
$$

He calls these $n$-tuples $C_{0}$ contractions, and provides a WOT-continuous functional calculus in [28]. We can analyze this using the theory of representations of $\mathfrak{L}_{n}$ developed in [16].

Let $A=\left(A_{1}, \ldots, A_{n}\right)$ be a $C_{0}$-contraction on $\mathcal{V}$ with pure minimal isometric dilation $S_{i} \simeq L_{i}^{(s)}$. This determines a WOT-continuous representation $\Phi_{A}$ of $\mathfrak{L}_{n}$ which sends $X$ to $\left.P_{\mathcal{V}} X^{(s)}\right|_{\mathcal{V}}$. In particular, $\Phi_{A}\left(L_{w}\right)=A_{w}:=A_{i_{1}} \ldots A_{i_{k}}$ for every word $w=i_{1} \ldots i_{k}$ in $\mathbb{F}_{n}^{+}$. The kernel $\mathfrak{J}=\operatorname{ker} \Phi_{A}$ is a WOT-closed ideal of $\mathfrak{L}_{n}$. By [16, Theorem 2.1], this ideal is determined by its range $\mathcal{M}=\overline{\mathfrak{J} \mathcal{K}_{n}}$, which is an invariant subspace for both $\mathfrak{L}_{n}$ and its commutant $\mathfrak{R}_{n}$. The representation of compression of $\mathfrak{L}_{n}$ to $\mathcal{M}^{\perp}$ has the same kernel. We wish to determine to what extent $A$ can be recovered from the compression of $L$ to $\mathcal{M}^{\perp}$.

To get a feeling for the situation, consider the case in which the $A_{i}$ are $d \times d$ matrices which generate $\mathfrak{M}_{d}$ as an algebra. Then $\Phi_{A}$ maps $\mathfrak{L}_{n}$ onto $\mathfrak{M}_{d}$. The kernel $\mathfrak{J}$ will then have codimension $d^{2}$, and therefore the dimension of $\mathcal{M}^{\perp}$ is also $d^{2}$. The compression homomorphism to $\mathcal{M}^{\perp}$ factors through $\Phi_{A}$. Since $\mathfrak{M}_{d}$ has only one irreducible representation up to similarity, the compression to $\mathcal{M}^{\perp}$ must be similar to the direct sum of $d$ copies of $\Phi_{A}$. In particular, $\mathcal{M}^{\perp}$ will decompose into a (nonorthogonal) direct sum of $d$ subspaces which are $\mathfrak{L}_{n}^{*}$-invariant such that the compression of $L$ is similar to $A$.

Nevertheless, $\Phi_{A}$ need not occur as a compression of $L$ to some $\mathfrak{L}_{n}^{*}$ invariant subspace. This could occur only if $\Phi_{A}$ has pure rank 1 , which need not be the case. However, this shows that there are representations similar to $\Phi_{A}$ which do have pure rank 1 . Moreover it turns out that in a certain sense, these similarities of pure rank 1 are the extreme points of those representations similar to $\Phi_{A}$. This will be established 
by showing that $\Phi_{A}$ can be recovered as a $C^{*}$-convex combination of pure rank 1 representations.

Theorem 7.6. Let $A=\left(A_{1}, \ldots, A_{n}\right)$ be a $C_{0}$-contraction on a $d$ dimensional space $\mathcal{V}$. Let $\mathfrak{J}$ be the kernel of the WOT-continuous representation $\Phi_{A}$ of $\mathfrak{L}_{n}$ that it determines. Then $\Phi_{A}$ is unitarily equivalent to the compression of $\mathfrak{L}_{n}$ to a semi-invariant subspace $\mathcal{S}=\mathcal{N}_{1} \ominus \mathcal{N}_{2}$, where $\mathcal{N}_{1}=\mathfrak{L}_{n}[\mathcal{S}]$ and $\mathcal{N}_{2}=\mathcal{N}_{1} \ominus \mathcal{S}$ belong to Lat $\mathfrak{L}_{n}$.

Let $\mathcal{M}=\overline{\mathfrak{J} \mathcal{K}_{n}}$ be the corresponding $\mathfrak{L}_{n}$ and $\mathfrak{R}_{n}$-invariant subspace associated to $\mathfrak{J}$. Then there are at most $d$ wandering vectors $\zeta_{j}$, say for $1 \leq j \leq s$ where $s \leq d$, with $\mathfrak{L}_{n}\left[\zeta_{i}\right]$ pairwise orthogonal, such that

$$
\mathcal{N}_{1}=\sum_{j=1}^{s} R_{\zeta_{j}} \mathcal{K}_{n} \quad \text { and } \quad \mathcal{N}_{2} \supset \sum_{j=1}^{s} R_{\zeta_{j}} \mathcal{M}
$$

Moreover, the subspaces $\mathcal{M}_{j}=R_{\zeta_{j}}^{*} \mathcal{S}$ are $\mathfrak{L}_{n}^{*}$-invariant subspaces of $\mathcal{M}^{\perp}$, and $\operatorname{dim}\left(\mathcal{M}_{j}\right) \leq d$. The contractive $n$-tuples $B_{j}=\left(B_{j 1}, \ldots, B_{j n}\right)$ obtained by compression of $L$ to $\mathcal{M}_{j}$ have pure rank 1 and $\operatorname{ker} \Phi_{B_{j}} \supset \mathfrak{J}$. There is an isometry $X$ mapping $\mathcal{S}$ into $\sum_{j=1}^{\oplus s} \mathcal{M}_{j}$ so that

$$
\left(\sum_{j=1}^{s} B_{j i}^{*}\right) X=X A_{i}^{*} \quad \text { for } \quad 1 \leq i \leq n .
$$

Thus $A^{*}$ is unitarily equivalent to the restriction of $\sum_{j=1}^{\oplus s} B_{j}^{*}$ to an invariant subspace. Consequently, $A=X^{*} \sum_{j=1}^{\oplus s} B_{j} X$ is a $C^{*}$-convex combination of the $B_{j}$ 's.

In particular, when $\mathfrak{A}=\mathcal{B}(\mathcal{V})$, each subspace $\mathcal{M}_{j}$ is d-dimensional and each $n$-tuple $B_{j}$ is similar to $A$.

Proof. The isometric dilation $S=\left(S_{1}, \ldots, S_{n}\right)$ of $A$ has pure rank $s=\operatorname{rank}\left(I-\sum_{i=1}^{n} A_{i} A_{i}^{*}\right) \leq d$. The identity representation of $\mathfrak{L}_{n}$ contains many invariant subspaces with infinite dimensional wandering space; and thus an infinite multiple of the identity representation is contained in $\mathfrak{L}_{n}$. So we may assume that $\Phi_{A}$ is the compression of $\mathfrak{L}_{n}$ to a semi-invariant subspace $\mathcal{S}$ of $\mathfrak{L}_{n}$ itself. The minimal choice of a pair of $\mathfrak{L}_{n}$-invariant subspaces with difference $\mathcal{S}$ is given by $\mathcal{N}_{1}=\mathfrak{L}_{n}[\mathcal{S}]$ and $\mathcal{N}_{2}=\mathcal{N}_{1} \ominus \mathcal{S}[33]$.

Now $\mathcal{N}_{1}$ has a wandering space $\mathcal{W}$ of dimension $s$. Choose an orthonormal basis $\zeta_{j}, 1 \leq j \leq s$, for $\mathcal{W}$. By [15, Theorem 2.1], there is an isometry $R_{\zeta_{j}}$ in $\mathfrak{R}_{n}$ with range equal to the cyclic $\mathfrak{L}_{n}$-invariant subspace $\mathfrak{L}_{n}\left[\zeta_{j}\right]$. Then $\mathcal{N}_{1}=\sum_{j=1}^{\oplus s} R_{\zeta_{j}} \mathcal{K}_{n}$. Since the kernel of the compression 
to $\mathcal{S}$ is $\mathfrak{J}$

$$
\begin{aligned}
\mathcal{N}_{2} & \supset \mathfrak{J} \mathcal{S}=\mathfrak{J} \mathfrak{L}_{n} \mathcal{S}=\mathfrak{J N}_{1} \\
& =\sum_{j=1}^{s} \mathfrak{J} R_{\zeta_{j}} \mathcal{K}_{n}=\sum_{j=1}^{s} R_{\zeta_{j}} \mathfrak{J} \mathcal{K}_{n}=\sum_{j=1}^{s} R_{\zeta_{j}} \mathcal{M} .
\end{aligned}
$$

The subspaces $\mathcal{M}_{j}=R_{\zeta_{j}}^{*} \mathcal{S}$ are contained in $\mathcal{M}^{\perp}$, and have dimension at most $d=\operatorname{dim} \mathcal{S}$. Moreover, they are $\mathfrak{L}_{n}^{*}$-invariant because of the identity

$$
\mathfrak{L}_{n}^{*} R_{\zeta_{j}}^{*} \mathcal{S}=R_{\zeta_{j}}^{*} \mathfrak{L}_{n}^{*} \mathcal{S} \subset R_{\zeta_{j}}^{*} \mathcal{N}_{2}^{\perp}=R_{\zeta_{j}}^{*} \mathcal{S}
$$

Let $B_{j}$ denote the contractive $n$-tuple obtained by compression of $L$ to $\mathcal{M}_{j}$. Clearly $L$ is an isometric dilation of $B_{j}$. The minimal dilation is obtained by restricting $L$ to $\mathfrak{L}_{n}\left[\mathcal{M}_{j}\right]$. However by Lemma 3.4, this is a reducing subspace of $\mathcal{K}_{n}$. Since the commutant $\mathfrak{R}_{n}$ of $\mathfrak{L}_{n}$ contains no idempotents [15, Corollary 1.8], this space must be all of $\mathcal{K}_{n}$. Thus the $n$-tuple $B_{j}$ has pure rank 1 for each $1 \leq j \leq s$. Since $\mathcal{M}_{j}$ is contained in $\mathcal{M}^{\perp}$, it follows that $\operatorname{ker} \Phi_{B_{j}}$ contains the ideal $\mathfrak{J}$.

Now notice that $R_{\zeta_{j}} \mathcal{M}_{j}$ are pairwise orthogonal subspaces, and

$$
\sum_{j=1}^{s} R_{\zeta_{j}} \mathcal{M}_{j}=\sum_{j=1}^{s} R_{\zeta_{j}} R_{\zeta_{j}}^{*} \mathcal{S} \supset\left(\sum_{j=1}^{s} R_{\zeta_{j}} R_{\zeta_{j}}^{*}\right) \mathcal{S}=P_{\mathcal{N}_{1}} \mathcal{S}=\mathcal{S} .
$$

This allows us to identify $\mathcal{S}$ isometrically with a subspace of $\sum_{j=1}^{\oplus s} \mathcal{M}_{j}$. Let $X_{j}=R_{\zeta_{j}}^{*} P_{\mathcal{S}}$ be considered as a map from $\mathcal{S}$ into $\mathcal{M}_{j}$. Define $X$ to be the column matrix $\left[\begin{array}{lll}X_{1} & \ldots & X_{s}\end{array}\right]^{t}$. Then

$$
X^{*} X=\sum_{j=1}^{s} P_{\mathcal{S}} R_{\zeta_{j}} R_{\zeta_{j}}^{*} P_{\mathcal{S}}=P_{\mathcal{S}} P_{\mathcal{N}_{1}} P_{\mathcal{S}}=P_{\mathcal{S}}
$$

So $X$ is an isometry of $\mathcal{S}$ into $\sum_{j=1}^{\oplus s} \mathcal{M}_{j}$. One may compute

$$
R_{\zeta_{j}}^{*} L_{i}^{*} P_{\mathcal{S}}=\left(R_{\zeta_{j}}^{*} P_{\mathcal{N}_{1}}\right)\left(P_{\mathcal{N}_{2}}^{\perp} L_{i}^{*} P_{\mathcal{S}}\right)=R_{\zeta_{j}}^{*} P_{\mathcal{S}} L_{i}^{*} P_{\mathcal{S}}
$$

Therefore identifying $A_{i}$ with $P_{\mathcal{S}} L_{i} P_{\mathcal{S}}$, we obtain

$$
\begin{aligned}
\left(\sum_{j=1}^{s} B_{j i}^{*}\right) X & =\sum_{j=1}^{s} L_{i}^{*} R_{\zeta_{j}}^{*} P_{\mathcal{S}}=\sum_{j=1}^{s} R_{\zeta_{j}}^{*} L_{i}^{*} P_{\mathcal{S}} \\
& =\sum_{j=1}^{s} R_{\zeta_{j}}^{*} P_{\mathcal{S}} L_{i}^{*} P_{\mathcal{S}}=X A_{i}^{*} .
\end{aligned}
$$

From this it is evident that the range of $X$ is invariant for $\sum_{j=1}^{\oplus s} B_{j i}^{*}$, and implements a unitary equivalence between $A_{i}^{*}$ and this restriction of $\sum_{j=1}^{\oplus s} B_{j i}^{*}$. Consequently, $X^{*}\left(\sum_{j=1}^{\oplus s} B_{j i} X\right)=A_{i}$ for $1 \leq i \leq n$. 
This expresses $A$ as a $\mathrm{C}^{*}$-convex combination of the pure rank one contractions $B_{j}$.

When $\mathfrak{A}$ is isomorphic to $\mathcal{B}(\mathcal{V}) \simeq \mathfrak{M}_{d}$, then $\mathfrak{L}_{n} / \mathfrak{J}$ is likewise isomorphic to $\mathfrak{M}_{d}$ and the compression of $\mathfrak{L}_{n}$ to $\mathcal{M}^{\perp}$ is a representation of $\mathfrak{M}_{d}$ on a subspace of dimension $d^{2}$. The only representations of $\mathfrak{M}_{d}$ are multiples of the identity representation up to similarity, and the compression to $\mathcal{M}^{\perp}$ has multiplicity $d$. Thus the $\mathfrak{L}_{n}^{*}$-invariant subspaces of $\mathcal{M}^{\perp}$ have dimension which is a multiple of $d$. As $\mathcal{M}_{j}$ are non-zero and have dimension at most $d$, they are all exactly $d$-dimensional and each $B_{j}^{*}$ is similar to $A^{*}$, whence $B_{j}$ is similar to $A$.

Say that the $n$-tuple $A$ is irreducible if it generates $\mathfrak{A}=\mathcal{B}(\mathcal{V})$, or equivalently $\mathfrak{A}$ has no proper invariant subspaces. When $A$ is an irreducible $C_{0}$-contraction, we see that the compression representation to $\mathcal{M}^{\perp}$ takes the generators to an $n$-tuple similar to the direct sum of $d$ copies of $A$. In particular, this occurs if $\|A\|<1$. So we obtain a complete similarity invariant for an arbitrary irreducible $n$-tuple of matrices (after scaling appropriately).

Restricting to the irreducible case is not just a matter of convenience. Simple examples show that multiplicity cannot be detected from the set of polynomial identities that an $n$-tuple satisfies. For example, with $n=1$, take $A=J_{2} \oplus 0^{(3)}$ and $B=J_{2}^{(2)} \oplus 0$ where $J_{2}$ is the $2 \times 2$ nilpotent Jordan matrix and 0 is a one-dimensional zero. These two matrices satisfy exactly the same polynomial identities. The natural way to distinguish them is to use rank. Indeed, familiar invariants for similarity of single matrices shows that the ranks of various polynomials can be used to determine the multiplicity function.

Corollary 7.7. Suppose that $A=\left(A_{1}, \ldots, A_{n}\right)$ and $B=\left(B_{1}, \ldots, B_{n}\right)$ are two $n$-tuples of $d \times d$ matrices which are irreducible and strictly contractive, $\|A\|<1$ and $\|B\|<1$. Then $A$ and $B$ are similar if and only if $\operatorname{ker} \Phi_{A}=\operatorname{ker} \Phi_{B}$.

Proof. Clearly two similar $n$-tuples give rise to representations with the same kernel. Conversely, if they are irreducible, the kernel determines the subspace $\mathcal{M}$. We adopt the notation from the proof of Theorem 7.6. A minimal $\mathfrak{L}_{n}^{*}$-invariant subspace $\mathcal{M}_{j}$ of $\mathcal{M}^{\perp}$ yields a compression representation $\Phi_{j}$ which is similar to $\Phi_{A}$ by Theorem 7.6. Likewise, $B$ determines the same subspaces, and thus $\Phi_{B}$ is also similar to $\Phi_{j}$, and hence to $\Phi_{A}$.

The similarity question for $n$-tuples of matrices is an old one, and the solution is complicated. Friedland [21] provides an algorithm for checking whether two $n$-tuples $A=\left(A_{1}, \ldots, A_{n}\right)$ and $B=\left(B_{1}, \ldots, B_{n}\right)$ 
of $d \times d$ matrices are similar. This is quite involved even for two $2 \times 2$ matrices, which he calculates explicitly. The situation simplifies when the two matrices are not simultaneously triangularizable - which in the $2 \times 2$ case is the same as irreducibility. In this case, the pairs $A$ and $B$ are similar if and only if these five identities hold:

$$
\begin{aligned}
\operatorname{Tr}\left(A_{1}\right) & =\operatorname{Tr}\left(B_{1}\right) & & \operatorname{Tr}\left(A_{1}^{2}\right)=\operatorname{Tr}\left(B_{1}^{2}\right) \\
\operatorname{Tr}\left(A_{2}\right) & =\operatorname{Tr}\left(B_{2}\right) & & \operatorname{Tr}\left(A_{2}^{2}\right)=\operatorname{Tr}\left(B_{2}^{2}\right) \\
\operatorname{Tr}\left(A_{1} A_{2}\right) & =\operatorname{Tr}\left(B_{1} B_{2}\right) . & &
\end{aligned}
$$

In general, there is no explicit list of polynomials to check.

In our case, we do obtain a fairly small finite list of invariants for an irreducible $n$-tuple. Unfortunately, at this point, we do not have an explicit method for computing these invariants. Nor are they polynomials. The natural invariants in our setting are isometries in $\mathfrak{L}_{n}$. Polynomials can be obtained by a simple approximation argument, but are no longer canonical. In the case of two $2 \times 2$ matrices, we obtain exactly five conditions.

Theorem 7.8. Let $A=\left(A_{1}, \ldots, A_{n}\right)$ be an irreducible $n$-tuple of $d \times d$ matrices with $\|A\|<1$. The ideal $\mathfrak{J}=\operatorname{ker} \Phi_{A}$ determines its range space $\mathcal{M}=\overline{\mathfrak{J} \mathcal{K}_{n}}$ with wandering dimension $1+(n-1) d^{2}$. Thus there are $1+(n-1) d^{2}$ isometries $X_{j}$ in $\mathfrak{L}_{n}$ so that an $n$-tuple $B$ of $d \times d$ matrices with $\|B\|<1$ is similar to $A$ if and only if $\Phi_{B}\left(X_{j}\right)=0$ for $1 \leq j \leq 1+(n-1) d^{2}$.

Moreover there is a set of $m=1+(n-1) d^{2}$ polynomials $p_{j}$ in $n$ non-commuting variables such that an $n$-tuple $B$ of $d \times d$ matrices with $\|B\|<1$ is similar to $A$ if and only if $p_{j}(B)=0$ for $1 \leq j \leq m$.

Proof. The space $\mathcal{M}$ has the same codimension as $\mathfrak{J}$, which is $d^{2}$ since $\mathfrak{L}_{n} / \mathfrak{J}$ is isomorphic to $\operatorname{Alg}\left\{A_{1}, \ldots, A_{n}\right\}=\mathfrak{M}_{d}$. Its wandering space is

$$
\begin{aligned}
\mathcal{W} & =\mathcal{M} \ominus \sum_{i=1}^{n} L_{i} \mathcal{M} \\
& =\mathcal{K}_{n} \ominus\left(\mathcal{M}^{\perp} \oplus \sum_{i=1}^{n} L_{i}\left(\mathcal{K}_{n} \ominus \mathcal{M}^{\perp}\right)\right) \\
& =\left(\left(\mathcal{K}_{n} \ominus \sum_{i=1}^{n} L_{i} \mathcal{K}_{n}\right) \oplus \sum_{i=1}^{n} L_{i} \mathcal{M}^{\perp}\right) \ominus \mathcal{M}^{\perp} \\
& =\left(\mathbb{C} \xi_{e} \oplus \sum_{i=1}^{n} L_{i} \mathcal{M}^{\perp}\right) \ominus \mathcal{M}^{\perp} .
\end{aligned}
$$

This has dimension $m=1+(n-1) \operatorname{dim} \mathcal{M}^{\perp}=1+(n-1) d^{2}$. 
Now $\mathcal{M}$ is invariant for both $\mathfrak{L}_{n}$ and its commutant $\mathfrak{R}_{n}$. Since it is the latter, it decomposes [15, Theorem 2.1] as the direct sum of $m$ cyclic $\mathfrak{R}_{n}$-invariant subspaces; and each is the range of an isometry $X_{j}$ in $\mathfrak{L}_{n}$. Thus by [16, Lemma 2.5], we obtain that $\mathfrak{J}=\sum_{j=1}^{m} X_{j} \mathfrak{L}_{n}$.

Therefore $\operatorname{ker} \Phi_{B}$ contains $\mathfrak{J}$ if and only if $\Phi_{B}\left(X_{j}\right)=0$ for $1 \leq j \leq$ $m$. Moreover, since $A$ is irreducible, $\mathfrak{J}$ is a maximal ideal. Thus this condition ensures that $\operatorname{ker} \Phi_{B}=\mathfrak{J}$. In particular, $\operatorname{Alg}\left\{B_{1}, \ldots, B_{n}\right\}$ is isomorphic to $\mathfrak{L}_{n} / \mathfrak{J} \simeq \mathfrak{M}_{d}$; and hence $B$ is also irreducible. Therefore $B$ and $A$ are similar by Corollary 7.7 .

To obtain polynomials, we notice that the algebra $\mathfrak{P}$ which is the algebraic span of $\left\{L_{w}: w \in \mathbb{F}_{n}^{+}\right\}$is WoT-dense in $\mathfrak{L}_{n}$. Let $\mathfrak{I}=\mathfrak{J} \cap \mathfrak{P}$ be the ideal of all polynomials which annihilate $A$. The algebra (without closure) generated by the $A_{i}$ 's is $\mathfrak{M}_{d}$. So the map $\Phi_{A}$ takes $\mathfrak{P}$ onto $\mathfrak{M}_{d}$ with kernel $\mathfrak{I}$; and takes $\mathfrak{L}_{n}$ onto $\mathfrak{M}_{d}$ with kernel $\mathfrak{J}$. It follows from the Hahn-Banach theorem that $\mathfrak{I}$ is wOT-dense in $\mathfrak{J}$.

Let $\varepsilon=\left(1+n d^{2}\right)^{-1}$. For each $1 \leq j \leq m$, choose polynomials $p_{j} \in \mathfrak{I}$ such that $\left\|p_{j}(L)-X_{j}\right\|<\varepsilon$. We claim that $p_{j}(L)$ generate $\mathfrak{J}$ as a norm-closed right ideal. For let $J \in \mathfrak{J}$. By [16, Lemma 2.5], there are elements $Y_{j} \in \mathfrak{L}_{n}$ such that $J=\sum_{j=1}^{m} X_{j} Y_{j}=: X Y$. Moreover, the row operator $X=\left[\begin{array}{lll}X_{1} & \ldots & X_{m}\end{array}\right]$ is an isometry. Hence the column operator $Y=\left[\begin{array}{lll}Y_{1} & \ldots & Y_{m}\end{array}\right]^{t}$ has $\|Y\|=\|J\|$. Let $P=\left[\begin{array}{llll}p_{1}(L) & \ldots & p_{m}(L)\end{array}\right]$. It follows that

$$
\|J-P Y\| \leq\|X-P\|\|Y\|<\frac{m}{1+n d^{2}}\|J\| .
$$

Since $m /\left(1+n d^{2}\right)<1$, the right ideal generated by $P$ is norm dense in $\mathfrak{J}$ as claimed.

Therefore the condition that $p_{j}(B)=0$ for $1 \leq j \leq m$ is equivalent to the condition $\Phi_{B}\left(X_{j}\right)=0$, and thus is equivalent to joint similarity to $A$.

While the $X_{j}$ 's are needed to generate $\mathfrak{J}$ as a WOT-closed right ideal, there will generally be redundancies as generators for $\mathfrak{J}$ as a two-sided ideal. So $1+(n-1) d^{2}$ is an upper bound on the number of test elements needed. It would be interesting to have better bounds on the number of generators for a two-sided ideal.

We observe that the existence of a determining set of polynomials for an irreducible $n$-tuple can be deduced directly by elementary means. One can write down polynomials in $A$ representing the matrix units of $d \times d$ matrices and their relations. In fact $O\left(d^{2}\right)$ generators and relations suffice. Then each $A_{i}$ can be expressed as a combination of matrix units. This requires only $n+O\left(d^{2}\right)$ polynomials, which is 
somewhat better than our bound. In many concrete cases, this simple bare hands approach is the best.

On the other hand, our result provides an algorithm for obtaining a set of generators for the ideal $\mathfrak{J}$. Perhaps this will prove to be of some use.

Example 7.9. This example illustrates parts of the previous two theorems. Consider the pair of $2 \times 2$ matrices

$$
A_{1}=\left[\begin{array}{cc}
0 & 1 / 2 \\
1 / 2 & 0
\end{array}\right] \quad \text { and } \quad A_{2}=\left[\begin{array}{cc}
1 / 2 & 0 \\
0 & 0
\end{array}\right]
$$

Since $I-A_{1} A_{1}^{*}-A_{2} A_{2}^{*}=\left[\begin{array}{cc}1 / 2 & 0 \\ 0 & 3 / 4\end{array}\right]$ has rank 2 , this determines a pure isometric dilation of pure rank 2 . The algebra $\mathfrak{A}=\mathfrak{M}_{2}$, and thus the representation $\Phi_{A}$ of $\mathfrak{L}_{2}$ is irreducible. The kernel will be a WOT-closed maximal ideal $\mathfrak{J}$ of codimension 4 . Therefore the subspace $\mathcal{M}=\overline{\mathfrak{J} \mathcal{K}_{2}}$ will be codimension 4 .

The matrices $A_{1}$ and $A_{2}$ satisfy certain relations that express the fact that

$$
\mathfrak{M}_{2}=\operatorname{Alg}\left\{A_{1}, A_{2}\right\}=\operatorname{span}\left\{I, A_{1}, A_{2}, A_{1} A_{2}\right\} .
$$

A natural and sufficient list is

(2) $\quad 2 A_{2}^{2}=A_{2}$

(3) $\quad A_{2} A_{1} A_{2}=0$

(4) $8 A_{1} A_{2} A_{1}=I-2 A_{2}$

(5) $\quad 2 A_{1} A_{2}+2 A_{2} A_{1}=A_{1}$

However, (5) and (2) can be derived from the others. So (1), (3) and (4) are sufficient.

The ideal $\mathfrak{J}$ is therefore generated as a two-sided ideal by the set

$$
\mathcal{J}=\left\{I-4 L_{1}^{2}, L_{2} L_{1} L_{2}, I-2 L_{2}-8 L_{1} L_{2} L_{1}\right\},
$$

since the quotient will be $\mathfrak{M}_{2}$. Therefore the range $\mathcal{M}$ is the $\mathfrak{L}_{2} \mathfrak{R}_{2^{-}}$ invariant subspace generated by $\mathcal{J} \xi_{e}$,

$$
\begin{aligned}
\mathcal{M} & =\overline{\mathfrak{J} \mathcal{K}_{2}}=\overline{\mathfrak{L}_{2} \mathfrak{R}_{2} \mathcal{J} \xi_{e}} \\
& =\operatorname{span}\left\{\xi_{u v}-4 \xi_{u 11 v}, \xi_{u 212 v}, \quad \xi_{u v}-2 \xi_{u 2 v}-8 \xi_{u 121 v}: u, v \in \mathbb{F}_{2}^{+}\right\} .
\end{aligned}
$$

We wish to determine $\mathcal{M}^{\perp}$. To this end, define $\Omega_{1}$ to be the set of all words in $11=1^{2}$ and 2 ,

$$
\Omega_{1}=\left\{w=1^{2 k_{0}} 21^{2 k_{1}} 2 \ldots 21^{2 k_{s}}: k_{i} \geq 0, s \geq 0\right\} .
$$


Let $\Omega_{2}=\Omega_{1} 1, \Omega_{3}=1 \Omega_{1}$ and $\Omega_{4}=\{e\} \cup 1 \Omega_{1} 1$. Define

$$
x_{i}=\sum_{w \in \Omega_{i}} 2^{-|w|} \xi_{w} \quad \text { for } \quad 1 \leq i \leq 4 .
$$

Then a computation shows that $\mathcal{M}^{\perp}=\operatorname{span}\left\{x_{1}, x_{2}, x_{3}, x_{4}\right\}$. These vectors are not orthogonal, nor of constant length. Indeed,

$$
\left\|x_{1}\right\|^{2}=16 / 11, \quad\left\|x_{2}\right\|^{2}=\left\|x_{3}\right\|^{2}=4 / 11 \quad \text { and } \quad\left\|x_{4}\right\|^{2}=12 / 11 .
$$

The pair $\left\{x_{1}, x_{4}\right\}$ is orthogonal to $\left\{x_{2}, x_{3}\right\}$, but

$$
\left(x_{1}, x_{4}\right)=16 / 15 \quad \text { and } \quad\left(x_{2}, x_{3}\right)=4 / 15 \text {. }
$$

Another matrix calculation relative to the ordered basis $\left\{x_{1}, \ldots, x_{4}\right\}$ for $\mathcal{M}^{\perp}$ shows that

$$
\left.L_{1}^{*}\right|_{\mathcal{M}^{\perp}}=\left[\begin{array}{cccc}
0 & 0 & 1 / 2 & 0 \\
0 & 0 & 0 & 1 / 2 \\
1 / 2 & 0 & 0 & 0 \\
0 & 1 / 2 & 0 & 0
\end{array}\right] \quad \text { and }\left.\quad L_{2}^{*}\right|_{\mathcal{M}^{\perp}}=\left[\begin{array}{cccc}
1 / 2 & 0 & 0 & 0 \\
0 & 1 / 2 & 0 & 0 \\
0 & 0 & 0 & 0 \\
0 & 0 & 0 & 0
\end{array}\right]
$$

Let $\Phi_{c}$ denote the representation of compression to $\mathcal{M}^{\perp}$. This calculation shows that $\Phi_{c}$ is similar (but not unitarily equivalent) to the direct sum of two copies of $\Phi_{A}$. Thus the compression to any two dimensional $\mathfrak{L}_{2}^{*}$-invariant subspace of $\mathcal{M}^{\perp}$ is similar to $\Phi_{A}$. As noted in the proof of Theorem 7.6, these representations all have pure rank 1. In particular, $\Phi_{A}$ does not occur as such a compression. It is also a fact that $\Phi_{c}$ is not unitarily equivalent to an orthogonal direct sum of two representations.

Next, we compute the 2-dimensional $\mathfrak{L}_{2}^{*}$-invariant subspaces of $\mathcal{M}^{\perp}$. Examining the representation $\Phi_{c}$, we observe that the two subspaces $\mathcal{M}_{1}:=\operatorname{span}\left\{x_{1}, x_{3}\right\}$ and $\mathcal{M}_{2}:=\operatorname{span}\left\{x_{2}, x_{4}\right\}$ are invariant for $\mathfrak{L}_{2}^{*}$. Setting $\eta_{i}=x_{i} /\left\|x_{i}\right\|$, we find that $\left\{\eta_{1}, \eta_{3}\right\}$ and $\left\{\eta_{2}, \eta_{4}\right\}$ are orthonormal bases for $\mathcal{M}_{1}$ and $\mathcal{M}_{2}$ respectively. Compute

$$
\begin{array}{rlrl}
\left.L_{1}^{*}\right|_{\mathcal{M}_{1}} \simeq B_{1}^{*} & =\left[\begin{array}{cc}
0 & 1 \\
1 / 4 & 0
\end{array}\right] & \text { and } & \left.L_{2}^{*}\right|_{\mathcal{M}_{1}} \simeq B_{2}^{*}=\left[\begin{array}{cc}
1 / 2 & 0 \\
0 & 0
\end{array}\right], \\
\left.L_{1}^{*}\right|_{\mathcal{M}_{2}} \simeq C_{1}^{*}=\left[\begin{array}{cc}
0 & 1 / \sqrt{12} \\
\sqrt{3} / 2 & 0
\end{array}\right] & \text { and } & \left.L_{2}^{*}\right|_{\mathcal{M}_{2}} \simeq C_{2}^{*}=\left[\begin{array}{cc}
1 / 2 & 0 \\
0 & 0
\end{array}\right] .
\end{array}
$$

These must be pairs which have pure rank 1 , as is verified by computing the ranks of

$$
I-B_{1} B_{1}^{*}-B_{2} B_{2}^{*}=\left[\begin{array}{cc}
11 / 16 & 0 \\
0 & 0
\end{array}\right] \quad \text { and } \quad I-C_{1} C_{1}^{*}-C_{2} C_{2}^{*}=\left[\begin{array}{cc}
0 & 0 \\
0 & 11 / 12
\end{array}\right] \text {. }
$$

The representation $\Phi_{c}$ factors through a representation of $\mathfrak{M}_{2}$ of multiplicity 2 . Thus every 2 -dimensional $\mathfrak{L}_{2}^{*}$-invariant subspace is the cyclic subspace determined by its intersection with the range of $\left.L_{2}^{*}\right|_{\mathcal{M}^{\perp}}=$ 
$\operatorname{span}\left\{\eta_{1}, \eta_{2}\right\}$, namely $\mathbb{C}\left(\alpha \eta_{1}+\beta \eta_{2}\right)$ for $|\alpha|^{2}+|\beta|^{2}=1$. The second vector spanning the subspace must be the image under $2 L_{1}^{*}$,

$$
2 L_{1}^{*}\left(\alpha \eta_{1}+\beta \eta_{2}\right)=2 \alpha B_{1}^{*} \eta_{1}+2 \beta C_{1}^{*} \eta_{2}=\frac{\alpha}{2} \eta_{3}+\sqrt{3} \beta \eta_{4} .
$$

A typical subspace of this form is

$$
\mathcal{M}_{\alpha, \beta}=\operatorname{span}\left\{\alpha \eta_{1}+\beta \eta_{2}, \frac{\alpha}{2} \eta_{3}+\sqrt{3} \beta \eta_{4}\right\} .
$$

However it is sufficient just to use $\mathcal{M}_{1}$ and $\mathcal{M}_{2}$, as they correspond to a particular choice of a basis for the wandering subspace of $\mathfrak{L}_{2}[\mathcal{S}]$.

Since rank considerations show that the subspace $\mathcal{S}$ cannot be $\mathfrak{L}_{2}^{*}$ invariant, we must write $\mathcal{S}$ as the difference of two $\mathfrak{L}_{2}$-invariant subspaces of multiplicity 2. We will look for an orthonormal set $\left\{\zeta_{1}, \zeta_{2}\right\}$ to span $\mathcal{S}$ of the form

$$
\zeta_{1}=\alpha R_{1} \eta_{1}+\beta R_{2} \eta_{2} \quad \text { and } \quad \zeta_{2}=\frac{\alpha}{2} R_{1} \eta_{3}+\sqrt{3} \beta R_{2} \eta_{4}
$$

They are always orthogonal, so the condition that they be norm one requires that

$$
|\alpha|^{2}+|\beta|^{2}=1=\frac{1}{4}|\alpha|^{2}+3|\beta|^{2} .
$$

This has the solution $|\alpha|^{2}=8 / 11$ and $|\beta|^{2}=3 / 11$. Therefore set

$$
\zeta_{1}=\sqrt{8 / 11} R_{1} \eta_{1}+\sqrt{3 / 11} R_{2} \eta_{2} \text { and } \zeta_{2}=\sqrt{2 / 11} R_{1} \eta_{3}+\sqrt{9 / 11} R_{2} \eta_{4}
$$

Another computation shows that

$$
\begin{aligned}
L_{1}^{*} \zeta_{1} & =\frac{1}{2} \zeta_{2}+\frac{1}{\sqrt{2}} \xi_{e} & L_{2}^{*} \zeta_{1} & =\frac{1}{2} \zeta_{1} \\
L_{1}^{*} \zeta_{2} & =\frac{1}{2} \zeta_{1} & L_{2}^{*} \zeta_{2} & =0+\frac{1}{2} \xi_{e}
\end{aligned}
$$

Thus we see that $\mathcal{S}:=\operatorname{span}\left\{\zeta_{1}, \zeta_{2}\right\}$ is a semi-invariant subspace $\mathcal{S}=$ $\mathcal{N}_{1} \ominus \mathcal{N}_{2}$ where $\mathcal{N}_{1}=\left\{\xi_{e}\right\}^{\perp}=\mathfrak{L}_{2}\left[\xi_{1}, \xi_{2}\right]$ and $\mathcal{N}_{2}=\left\{\xi_{e}, \zeta_{1}, \zeta_{2}\right\}^{\perp}$. Moreover these identities show that the compression of $L_{i}$ to $\mathcal{S}$ is unitarily equivalent to $A_{i}$ for $i=1,2$.

Let us compute the operators $X_{1}$ and $X_{2}$ promised in Theorem 7.6. The projection onto $\mathcal{S}$ is given by $P_{\mathcal{S}}=\zeta_{1} \zeta_{1}^{*}+\zeta_{2} \zeta_{2}^{*}$. Then

$$
X_{1}=R_{1}^{*} P_{\mathcal{S}}=\sqrt{\frac{8}{11}} \eta_{1} \zeta_{1}^{*}+\sqrt{\frac{2}{11}} \eta_{3} \zeta_{2}^{*}
$$

and

$$
X_{2}=R_{2}^{*} P_{\mathcal{S}}=\sqrt{\frac{3}{11}} \eta_{2} \zeta_{1}^{*}+\sqrt{\frac{9}{11}} \eta_{4} \zeta_{2}^{*}
$$


So recalling the matrix forms for $B_{i}$ and $C_{i}$, we obtain

$$
\begin{aligned}
\sum_{i=1}^{2} X_{i}^{*} L_{1} X_{i}= & {\left[\begin{array}{cc}
\sqrt{8 / 11} & 0 \\
0 & \sqrt{2 / 11}
\end{array}\right]\left[\begin{array}{cc}
0 & 1 / 4 \\
1 & 0
\end{array}\right]\left[\begin{array}{cc}
\sqrt{8 / 11} & 0 \\
0 & \sqrt{2 / 11}
\end{array}\right] } \\
& +\left[\begin{array}{cc}
\sqrt{3 / 11} & 0 \\
0 & \sqrt{9 / 11}
\end{array}\right]\left[\begin{array}{cc}
0 & \sqrt{3} / 2 \\
1 / \sqrt{12} & 0
\end{array}\right]\left[\begin{array}{cc}
\sqrt{3 / 11} & 0 \\
0 & \sqrt{9 / 11}
\end{array}\right] \\
= & {\left[\begin{array}{cc}
0 & 1 / 11 \\
4 / 11 & 0
\end{array}\right]+\left[\begin{array}{cc}
0 & 9 / 22 \\
3 / 22 & 0
\end{array}\right]=\left[\begin{array}{cc}
0 & 1 / 2 \\
1 / 2 & 0
\end{array}\right]=A_{1} }
\end{aligned}
$$

and

$$
\begin{aligned}
\sum_{i=1}^{2} X_{i}^{*} L_{2} X_{i}= & {\left[\begin{array}{cc}
\sqrt{8 / 11} & 0 \\
0 & \sqrt{2 / 11}
\end{array}\right]\left[\begin{array}{cc}
1 / 2 & 0 \\
0 & 0
\end{array}\right]\left[\begin{array}{cc}
\sqrt{8 / 11} & 0 \\
0 & \sqrt{2 / 11}
\end{array}\right] } \\
& +\left[\begin{array}{cc}
\sqrt{3 / 11} & 0 \\
0 & \sqrt{9 / 11}
\end{array}\right]\left[\begin{array}{cc}
1 / 2 & 0 \\
0 & 0
\end{array}\right]\left[\begin{array}{cc}
\sqrt{3 / 11} & 0 \\
0 & \sqrt{9 / 11}
\end{array}\right] \\
= & {\left[\begin{array}{cc}
4 / 11 & 0 \\
0 & 0
\end{array}\right]+\left[\begin{array}{cc}
3 / 22 & 0 \\
0 & 0
\end{array}\right]=\left[\begin{array}{cc}
1 / 2 & 0 \\
0 & 0
\end{array}\right]=A_{2} . }
\end{aligned}
$$

The wandering space for $\mathcal{M}$ has dimension 5 , and as in the proof of Theorem 7.8 is given by

$$
\mathcal{W}=\operatorname{span}\left\{\xi_{e}, L_{1} x_{j}, L_{2} x_{j}: 1 \leq j \leq 4\right\} \ominus \operatorname{span}\left\{x_{j}: 1 \leq j \leq 4\right\} .
$$

We do not compute a basis for this space, as the result is not particularly illuminating. But such a basis corresponds to 5 isometries $X_{1}, \ldots, X_{5}$ in $\mathfrak{L}_{n}$ such that $\mathfrak{J}=\sum_{j=1}^{5} X_{j} \mathfrak{L}_{n}$. Thus $\operatorname{ker} \Phi_{B}=\mathfrak{J}$ if and only if $\Phi_{B}\left(X_{j}\right)=0$ for $1 \leq j \leq 5$. But as we noted earlier in our remarks, finding generators for $\mathfrak{J}$ as a right ideal is overkill. It suffices to use generators for $\mathfrak{J}$ as a two sided ideal. Thus it is sufficient to verify the 3 polynomial conditions:

$$
\begin{aligned}
& 4 B_{1}^{2}=I \\
& B_{2} B_{1} B_{2}=0 \\
& 8 B_{1} B_{2} B_{1}=I-2 B_{2}
\end{aligned}
$$

It is an easy exercise to verify directly that these conditions suffice to determine the pair up to similarity.

Pursuing this example further, let us consider other contractive pairs which are similar to $A$. A calculation shows that these pairs are unitarily equivalent to a pair of the form

$$
\widetilde{A}_{1}=\left[\begin{array}{cc}
-a t & \frac{1}{4 t}-a^{2} t \\
t & a t
\end{array}\right] \quad \widetilde{A}_{2}=\left[\begin{array}{cc}
1 / 2 & a / 2 \\
0 & 0
\end{array}\right] \quad \text { where } t>0 \text { and } a \in \mathbb{C} \text {. }
$$


Since this is a contractive pair, $Z:=I-\widetilde{A}_{1} \widetilde{A}_{1}^{*}-\widetilde{A}_{2} \widetilde{A}_{2}^{*} \geq 0$. It suffices to check that $Z_{22} \geq 0$ and $\operatorname{det} Z \geq 0$. In other words,

$$
\begin{aligned}
& \left(1+|a|^{2}\right) t^{2} \leq 1 \quad \text { and } \\
& 12\left(1+|a|^{2}\right)^{2} t^{4}-\left(13-4|a|^{2}+8 \operatorname{Re} a^{2}\right) t^{2}+1 \leq 0 .
\end{aligned}
$$

The dilation of this pair has pure rank 1 if and only if the determinant is 0 , which requires an equality in (2). Notice that when $a=0$, one obtains the inequality $1 / \sqrt{12} \leq t \leq 1$. The extremes yield the two pure index 1 pairs $\left(B_{1}, B_{2}\right)$ and $\left(C_{1}, C_{2}\right)$ obtained above.

\section{REFERENCES}

[1] A. Arias and G. Popescu, Factorization and reflexivity on Fock spaces, Int. Equat. Oper. Th. 23 (1995), 268-286.

[2] W. Arveson, Interpolation problems in nest algebras, J. Func. Anal. 20 (1975), 208-233.

[3] W. Arveson, Subalgebras of $C^{*}$-algebras III: multivariable operator theory, Acta Math., to appear.

[4] W. Arveson, The curvature invariant of a Hilbert module over $\mathbb{C}\left[z_{1}, \ldots, z_{n}\right]$, preprint, 1998.

[5] E. Azoff, C.K. Fong and F. Gilfeather, A reduction theory for nonself-adjoint operator algebras, Trans. Amer. Math. Soc. 224 (1976), 351-366.

[6] H. Bercovici, Hyper-reflexivity and the factorization of linear functionals, J. Func. Anal. 158 (1998), 242-252.

[7] A. Beurling, On two problems concerning linear transformations in Hilbert space, Acta Math. 81 (1949), 239-255.

[8] O. Bratteli, P. Jorgensen, and G.L. Price, Endomorphisms of $\mathcal{B}(\mathcal{H})$, in "Quantization of nonlinear partial differential equations" (W. Arveson et al, Eds.), Proc. Symp. Pure Math. 59 pp. 93-138, Amer. Math. Soc., Providence, 1996.

[9] O. Bratteli and P. Jorgensen, Endomorphisms of $\mathcal{B}(\mathcal{H})$ II, J. Func. Anal. 145 (1997), 323-373.

[10] O. Bratteli and P. Jorgensen, Iterated function systems and permutation representations of the Cuntz algebra, Mem. Amer. Math. Soc., to appear.

[11] J. Bunce, Models for n-tuples of non-commuting operators, J. Func. Anal. 57 (1984), 21-30.

[12] E. Christensen, Perturbations of operator algebras II, Indiana U. Math. J. 26 (1977), 891-904.

[13] J. Cuntz, Simple $C^{*}$-algebras generated by isometries, Comm. Math. Phys. 57 (1977), 173-185.

[14] K.R. Davidson, The distance to the analytic Toeplitz operators, Illinois J. Math. 31 (1987), 265-273.

[15] K.R. Davidson and D.R. Pitts, Invariant subspaces and hyper-reflexivity for free semi-group algebras, Proc. London Math. Soc. 78 (1999), 401-430.

[16] K.R. Davidson and D.R. Pitts, The algebraic structure of non-commutative analytic Toeplitz algebras, Math. Ann. 311 (1998), 275-303.

[17] K.R. Davidson and D.R. Pitts, Nevanlinna-Pick Interpolation for noncommutative analytic Toeplitz algebras, Integral Eqtns. \& Operator Thy. 31 (1998), 321-337. 
[18] J. Dyer, A. Pedersen and P. Porcelli, An equivalent formulation of the invariant subspace problem, Bull. Amer. Math. Soc. 78 (1972), 1020-1023.

[19] A. Frahzo, Models for non-commuting operators, J. Func. Anal. 48 (1982), $1-11$.

[20] A. Frahzo, Complements to models for non-commuting operators, J. Func. Anal. 59 (1984), 445-461.

[21] S. Friedland, Simultaneous similarity of matrices, Adv. Math. 50 (1983), 189265.

[22] J. Glimm, Type I $C^{*}$-algebras, Ann. Math. 73 (1961), 572-612.

[23] D.W. Kribs, Factoring in non-commutative analytic Toeplitz algebras, J. Operator Theory. to appear.

[24] D.W. Kribs, The curvature invariant of a non-commuting n-tuple, preprint, 2000.

[25] M. Laca, Endomorphisms of $\mathcal{B}(\mathcal{H})$ and Cuntz algebras, J. Operator Theory 30 (1993), 381-396.

[26] G. Popescu, Isometric dilations for infinite sequences of noncommuting operators, Trans. Amer. Math. Soc. 316 (1989), 523-536.

[27] G. Popescu, Multi-analytic operators and some factorization theorems, Indiana Univ. Math. J. 38 (1989), 693-710.

[28] G. Popescu, Von Neumann Inequality for $\left(\mathcal{B}(\mathcal{H})^{n}\right)_{1}$, Math. Scand. 68 (1991), 292-304.

[29] G. Popescu, Multi-analytic operators on Fock spaces, Math. Ann. 303 (1995), 31-46.

[30] G. Popescu, Poisson transforms on some $C^{*}$-algebras generated by isometries, J. Func. Anal., to appear.

[31] R. Powers, An index theory for semigroups of $*$-endomorphisms of $\mathcal{B}(\mathcal{H})$ and type II factors, Can. J. Math. 40 (1988), 86-114.

[32] H. Radjavi and P. Rosenthal, Invariant subspaces, Ergebnisse der Math. und ihrer Grenz. 77. New York-Heidelberg-Berlin: Springer-Verlag, 1973.

[33] D. Sarason, On spectral sets having connected complement, Acta Sci. Math. (Szeged) 26 (1965), 289-299.

[34] B. Sz. Nagy,and C. Foiaş, Harmonic analysis of operators on Hilbert space, North Holland Pub. Co., London, 1970.

[35] J. Wermer, On invariant subspaces of normal operators, Proc. Amer. Math. Soc. 3 (1952), 270-277.

Pure Math. Dept., U. Waterloo, Waterloo, On N2L 3G1, CANAdA

E-mail address: krdavids@uwaterloo.ca

Pure Math. Dept., U. Waterloo, Waterloo, On N2L 3G1, CANAdA

E-mail address: dwkribs@uwaterloo.ca

Mitra Imaging Inc., 455 Phillip St., Waterloo, ON N2L 1W3, Canada

E-mail address: mshpigel@mitra.com 